\title{
Title: Decreased torsinA or LINC Complex Function Rescues a Laminopathy in Caenorhabditis elegans
}

\author{
Authors: Gabriela Huelgas-Morales ${ }^{1}$, Mark Sanders², Gemechu Mekonnen ${ }^{1}$, Tatsuya \\ Tsukamoto $^{1}$, David Greenstein ${ }^{1 *}$.
}

\begin{abstract}
Affiliations: ${ }^{1}$ Department of Genetics, Cell Biology, and Development, University of Minnesota, Minneapolis, MN 55455 USA. ${ }^{2}$ University Imaging Center, University of Minnesota, Minneapolis, MN 55455 USA. *Correspondence to: David Greenstein. Email: green959@umn.edu.
\end{abstract}

Abstract: The function of the nucleus depends on the integrity of the nuclear lamina, an intermediate filament network associated with the linker of nucleoskeleton and cytoskeleton (LINC) complex spanning the nuclear envelope. In turn, the AAA+ATPase torsinA regulates force transmission from the cytoskeleton to the nucleus. In humans, mutations affecting nuclear envelope-associated proteins cause laminopathies, including progeria, myopathy, and dystonia. We report that decreasing the function of the C. elegans torsinA homolog, OOC-5, rescues the sterility and premature aging caused by a null mutation in the single worm lamin homolog, $\operatorname{lmn}-1$. Loss of OOC-5 activity prevents nuclear collapse in $l m n-1$ mutants by disrupting the function of the LINC complex. These results suggest that LINC complex-transmitted forces damage nuclei with a compromised nuclear lamina.

One Sentence Summary: Inhibiting LINC complex activity prevents a progeric syndrome in $C$. elegans. 
Main Text: Deleterious mutations in many conserved nuclear envelope (NE) components cause human diseases (1). Despite the widespread expression of NE components, these diseases often cause tissue-specific pathologies. For example, mutations affecting the NE-associated AAA+ ATPase torsinA, which is expressed in most cell types, cause early-onset DYT1 dystonia and congenital joint contractures (2-4). In the nematode C. elegans, mutations in the torsinA ortholog, OOC-5, cause oocyte growth and embryonic polarity defects $(5,6)$. The cellular functions of torsin A appear to be conserved across cell types and species because distantly related organisms lacking torsinA function display similar ultrastructural NE defects $(7,8)$. Nonetheless, the molecular interactions by which torsinA carries out its cellular functions remain enigmatic.

To address the mechanism of OOC-5 function, we purified OOC-5-associated proteins from soluble and membrane protein fractions using tandem affinity purification (fig. S1 and table S1). Both purifications identified highly conserved regulators of endoplasmic reticulum (ER) and NE homeostasis (table S1). The OOC-5-associated proteins include OOC-3, a protein required for OOC-5 localization and function $(5,6)$, ER chaperone CNX-1/calnexin, which associates with torsinA in mammalian cells (9), and GEI-18 and T09B4.2, the divergent C. elegans homologs of the torsinA-interacting proteins LAP1 and LULL1 (10). Also among the OOC-5-associated proteins was SUN-1, the SUN domain inner nuclear membrane protein component of LINC complexes in the C. elegans germline (11). SUN domain proteins interact with nuclear lamins (12, 13), and the sole C. elegans lamin, LMN-1 (14), was well represented among OOC-5-associated proteins (42-43\% coverage). The genetic interaction studies described below suggest that the association between OOC-5, which resides in the lumen of the NE and ER, and nucleoplasmic LMN-1 is likely mediated through SUN-1. 
To address the significance of the protein associations with OOC-5, we conducted genetic analyses. The most striking result was an interaction between ooc-5 and $l m n-1$. Most $l m n-1$ null mutants are agametic, sterile, and have small gonads (Fig. 1B and Fig. 2B). However, $12.3 \pm 7.8 \%$ of animals ("escapers") develop larger gonads and produce dead embryos (Fig. 1C and Fig. 2B). Surprisingly, compromising ooc-5 function rescued the sterility of $l m n-1$ mutants such that all animals produced gametes and dead embryos (Fig. 1D, Fig. 2, C and D, and fig. S2). Importantly, reducing the dosage of ooc-5 was sufficient to suppress $l m n-1$ sterility. Reducing or eliminating ooc-5 function also suppressed the progeric defects (Fig. 1E) previously described in lmn-1(-) mutants (15).

The germ cells of the $l m n-1$ escapers appeared to deteriorate as they progressed through meiotic prophase; they assembled a synaptonemal complex (SC) but their nuclei collapse soon after the pachytene stage (fig. S3). SUN-1 lost its NE localization and appeared diffuse in most collapsing nuclei (fig. S4). Diakinesis-stage chromosomes were rare in $l m n-1$ mutant oocytes but were observed in most or all lmn-1 mutants after reducing ooc-5 function (Fig. 1 and fig. S3). Decreasing ooc-5 function did not appear to rescue the elevated levels of DNA damage observed in the late-stage pachytene female germ cells of lmn-1 mutants (fig. S5).

Studies in mammalian cells show that torsinA regulates force transmission from the cytoplasm to the nucleus (16). Thus we examined a model in which OOC-5 promotes LINC complex function, which damages nuclei with a compromised nuclear lamina. We tested for genetic interactions between lmn-1(-) mutants and genes encoding components of the LINC complex, utilizing mutations in sun-1 and $z y g-12$, which encodes the KASH domain outer nuclear membrane LINC complex-component in the germline (11). As observed for ooc-5, the proportion of fertile lmn-1 animals increased when the LINC complex function was compromised, and nuclear collapse was substantially ameliorated (Fig. 2, E-J, fig. S2, and fig. S6). These results 
suggest that OOC-5 and the LINC complex-transmitted forces contribute to nuclear damage in lmn-1 mutants.

To assess whether OOC-5 promotes LINC complex assembly, we used super-resolution microscopy to examine the colocalization of SUN-1 and ZYG-12 in the wild type and ooc-5 mutants. Prior work showed discontinuities in ZYG-12 localization in ooc-5 mutants commencing in pachytene germ cells (8). In addition to these discontinuities, we observed a reduction of ZYG$12 \mathrm{NE}$ localization as germ cells progressed from the pachytene stage to the diakinesis stage of meiotic prophase (Fig. 2L). By contrast, SUN-1 remained in the NE at high levels in ooc-5 mutants (Fig. 2L). We quantified SUN-1 and ZYG-12 colocalization as a function of nuclear diameter and observed that as the germ cells grew, colocalization declined (Fig. 2, M and N). These results suggest that OOC-5 promotes the assembly or maintenance of the LINC complex, which appears particularly important for growing meiotic prophase nuclei.

The LINC complex is essential for the pairing of homologous chromosomes in C. elegans meiosis (17-19). OOC-5 is not required for SC formation (fig. S3) and appears dispensable for meiotic chromosome segregation (5). To examine whether OOC-5 promotes the efficiency of meiotic pairing, we examined the localization of ZIM-3 and HIM-8, which bind the pairing center regions on chromosomes I and IV and the X chromosome, respectively (20). The spatial distribution of unpaired meiotic chromosomes across the distal gonad suggests that pairing of chromosomes I and IV is delayed in ooc-5 mutants (Fig. 3B). By contrast, ooc-5 appears dispensable for the timely pairing of the $\mathrm{X}$ chromosome (Fig. 3C). Persistent SUN-1 phosphorylation correlates with synaptic or recombination defects (21). We observed that phosphorylated SUN-1 (S8Pi) persisted in an extended zone in ooc-5 mutants (Fig. 3, D-F), and the number of SUN-1 foci and their speed of motion increased (Fig. 3, G and H, and mov. S1-3). 
The increased movement of $\mathrm{SUN}-1$ foci in ooc-5 mutants may reflect partial release from cytoskeletal tension. These results suggest that the function of the LINC complex is perturbed in ooc-5 mutants even in early prophase germ cells, where SUN-1 and ZYG-12 colocalize normally.

C. elegans OOC-5 and mammalian torsinA are required for the proper localization of nuclear pore complex (NPC) proteins $(8,22)$. Mislocalization of nucleoporins (Nups) may be sufficient to explain the embryonic polarity defects in ooc-5 mutants (23). The two transmembrane Nups, NPP-12 and NPP-22, were identified among OOC-5-associated proteins (table S1). A prior study concluded that an NPP-22::GFP-expressing transgene localized more normally in ooc-5 mutants than other Nups, but the localization of NPP-12 was not examined (8). Thus, we used genome editing to generate GFP fusions to the endogenous proteins and analyzed their localization. Both NPP-12::GFP and GFP::NPP-22 exhibited substantial mislocalization in ooc-5 mutants, accumulating largely outside the NE and in the cytoplasm of proximal oocytes (Fig. 4, B, D, and G). This mislocalization of Nups in ooc-5 mutants might be a consequence of the impaired function of the LINC complex, as prior work showed that Sun1 disruption in mammalian cells lead to NPC mislocalization (24). Consistent with this possibility, NPP-12::GFP exhibited mislocalization in sun-1 and zyg-12 mutants (Fig. 4, E-G).

Our results indicate that OOC-5 promotes LINC complex functions needed to maintain NE homeostasis. Further, these data suggest that in the absence of a functional nuclear lamina, LINC complex-dependent forces promoted by OOC-5 result in nuclear damage (Fig. 4H). Consistent with this model, disruptions of the LINC complex can ameliorate defects in cultured cell and mouse models of Hutchinson-Gilford progeria (25). Also, LINC complex-dependent forces may be important for maintaining nuclear structure and protecting the NE from mechanical damage, as suggested by a study of fibroblasts from DYT1 dystonia patients (26). Age-related alterations in 
the ability to cope with mechanotransduction across the NE may contribute to progeric syndromes and reduce normal healthspan.

\section{References and Notes:}

1. A. Janin, D. Bauer, F. Ratti, G. Millat, A. Méjat, Nuclear envelopathies: a complex LINC between nuclear envelope and pathology. Orphanet J Rare Dis 12, 147 (2017).

2. L. J. Ozelius, W. Hewett, C. E. Page, S. B. Bressman, P. L. Kramer, C. Shalish, D. de Leon, M. F. Brin, D. Raymond, D. P. Corey, S. Fahn, N. J. Risch, A. J. Buckler, J. F. Gusella, X. O. Breakefield, The early-onset torsion dystonia gene (DYT1) encodes an ATP-binding protein. Nat Genet 17, 40-48 (1997).

3. A. Kariminejad, M. Dahl-Halvarsson, G. Ravenscroft, F. Afroozan, E. Keshavarz, H. Goulleé, M. R. Davis, M. F. Zonooz, H. Najmabadi, N. G. Laing, H. Tajsharghi, TOR1A variants cause a severe arthrogryposis with developmental delay, strabismus and tremor. Brain 140, 2851-2859 (2017).

4. S. C. Reichert, P. Gonzalez-Alegre, G. H. Scharer, Biallelic TOR 1A variants in an infant with severe arthrogryposis. Neurol Genet 3, e154 (2017).

5. S. E. Basham, L. S. Rose, Mutations in ooc-5 and ooc-3 disrupt oocyte formation and the reestablishment of asymmetric PAR protein localization in two-cell Caenorhabditis elegans embryos. Dev Biol 215, 253-263 (1999).

6. S. E. Basham, L. S. Rose, The Caenorhabditis elegans polarity gene ooc-5 encodes a Torsin-related protein of the AAA ATPase superfamily. Development 128, 4645-4656 (2001). 
7. R. E. Goodchild, C. E. Kim, W. T. Dauer, Loss of the dystonia-associated protein torsinA selectively disrupts the neuronal nuclear envelope. Neuron 48, 923-932 (2005).

8. M. J. VanGompel, K. C. Nguyen, D. H. Hall, W. T. Dauer, L. S. Rose, A novel function for the Caenorhabditis elegans torsin OOC-5 in nucleoporin localization and nuclear import. Mol Biol Cell 26, 1752-1763 (2015).

9. T. V. Naismith, S. Dalal, P. I. Hanson, Interaction of torsinA with its major binding partners is impaired by the dystonia-associated DeltaGAG deletion. J Biol Chem 284, 27866-27874 (2009).

10. A. E. Rose, R. S. H. Brown, C. Schlieker, Torsins: not your typical AAA+ ATPases. Crit Rev Biochem Mol Biol 50, 532-549 (2015).

11. I. L. Minn, M. M. Rolls, W. Hanna-Rose, C. J. Malone, SUN-1 and ZYG-12, mediators of centrosome-nucleus attachment, are a functional SUN/KASH pair in Caenorhabditis elegans. Mol Biol Cell 20, 4586-4595 (2009).

12. M. Crisp, Q. Liu, K. Roux, J. B. Rattner, C. Shanahan, B. Burke, P. D. Stahl, D. Hodzic, Coupling of the nucleus and cytoplasm: role of the LINC complex. J Cell Biol 172, 41-53 (2006).

13. C. R. Bone, E. C. Tapley, M. Gorjánácz, D. A. Starr, The Caenorhabditis elegans SUN protein UNC-84 interacts with lamin to transfer forces from the cytoplasm to the nucleoskeleton during nuclear migration. Mol Biol Cell 25, 2853-2865 (2014).

14. J. Liu, T. Rolef Ben-Shahar, D. Riemer, M. Treinin, P. Spann, K. Weber, A. Fire, Y. Gruenbaum, Essential roles for Caenorhabditis elegans lamin gene in nuclear organization, cell cycle progression, and spatial organization of nuclear pore complexes. Mol Biol Cell 11, 3937-3947 (2000). 
15. E. Haithcock, Y. Dayani, E. Neufeld, A. J. Zahand, N. Feinstein, A. Mattout, Y. Gruenbaum, J. Liu, Age-related changes of nuclear architecture in Caenorhabditis elegans. Proc Natl Acad Sci U S A 102, 16690-16695 (2005).

16. C. A. Saunders, N. J. Harris, P. T. Willey, B. M. Woolums, Y. Wang, A. J. McQuown, A. Schoenhofen, H. J. Worman, W. T. Dauer, G. G. Gundersen, G. W. G. Luxton, TorsinA controls TAN line assembly and the retrograde flow of dorsal perinuclear actin cables during rearward nuclear movement. J Cell Biol 216, 657-674 (2017).

17. A. Penkner, L. Tang, M. Novatchkova, M. Ladurner, A. Fridkin, Y. Gruenbaum, D. Schweizer, J. Loidl, V. Jantsch, The nuclear envelope protein Matefin/SUN-1 is required for homologous pairing in C. elegans meiosis. Dev Cell 12, 873-885. (2007).

18. A. Sato, B. Isaac, C. M. Phillips, R. Rillo, P. M. Carlton, D. J. Wynne, R. A. Kasad, A. F. Dernburg, Cytoskeletal forces span the nuclear envelope to coordinate meiotic chromosome pairing and synapsis. Cell 139, 907-919 (2009).

19. A. M. Penkner, A. Fridkin, J. Gloggnitzer, A. Baudrimont, T. Machacek, A. Woglar, E. Csaszar, P. Pasierbek, G. Ammerer, Y. Gruenbaum, V. Jantsch, Meiotic chromosome homology search involves modifications of the nuclear envelope protein Matefin/SUN-1. Cell 139, 920-933 (2009).

20. O. Rog, A. F. Dernburg, Chromosome pairing and synapsis during Caenorhabditis elegans meiosis. Curr Opin Cell Biol, 349-356 (2013).

21. A. Woglar, A. Daryabeigi, A. Adamo, C. Habacher, T. Machacek, A. La Volpe, V. Jantsch, Matefin/SUN-1 phosphorylation is part of a surveillance mechanism to coordinate chromosome synapsis and recombination with meiotic progression and chromosome movement. PLoS Genet 9, e1003335 (2013). 
22. S. S. Pappas, C. C. Liang, S. Kim, C. O. Rivera, W. T. Dauer, TorsinA dysfunction causes persistent neuronal nuclear pore defects. Hum Mol Genet 27, 407-420 (2018).

23. A. Schetter, P. Askjaer, F. Piano, I. Mattaj, K. Kemphues, Nucleoporins NPP-1, NPP-3, NPP4, NPP-11, and NPP-14 are required for proper spindle orientation in C. elegans. Dev Biol 289, 360-371 (2006).

24. Q. Liu, N. Pante, T. Misteli, M. Elsagga, M. Crisp, D. Hodzic, B. Burke, K. J. Roux, Functional association of Sun1 with nuclear pore complexes. J Cell Biol 178, 785-798 (2007).

25. P. H. Kim, J. Luu, P. Heizer, Y. Tu, T. A. Weston, N. Chen, C. Lim, R. L. Li, P. Y. Lin, J. C. Y. Dunn, D. Hodzic, S. G. Young, L. G. Fong, Disrupting the LINC complex in smooth muscle cells reduces aortic disease in a mouse model of Hutchinson-Gilford progeria syndrome. Sci Transl Med 10, (2018).

26. N. K. Gill, C. Ly, P. H. Kim, C. A. Saunders, L. G. Fong, S. G. Young, G. W. G. Luxton, A. C. Rowat, DYT1 dystonia patient-derived fibroblasts have increased deformability and susceptibilty to damage by mechanical forces. Front Cell Dev Biol 7, 103 (2019).

27. J. A. Arribere, R. T. Bell, B. X. Fu, K. L. Artiles, P. S. Hartman, A. Z. Fire, Efficient marker-free recovery of custom genetic modifications with CRISPR/Cas9 in Caenorhabditis elegans. Genetics 198, 837-846 (2014).

28. D. J. Dickinson, J. D. Ward, D. J. Reiner, B. Goldstein, Engineering the Caenorhabditis elegans genome using Cas9-triggered homologous recombination. Nat Methods 10, 1028-1034 (2013).

29. A. Paix, Y. Wang, H. E. Smith, C. Y. Lee, D. Calidas, T. Lu, J. Smith, H. Schmidt, M. W. Krause, G. Seydoux, Scalable and versatile genome editing using linear DNAs with 
microhomology to Cas9 Sites in Caenorhabditis elegans. Genetics 198, 1347-1356 (2014).

30. D. J. Dickinson, A. M. Pani, J. K. Heppert, C. D. Higgins, B. Goldstein, Streamlined genome engineering with a self-excising drug selection cassette. Genetics 200, 10351049 (2015).

31. C. A. Spike, D. Coetzee, Y. Nishi, T. Guven-Ozkan, M. Oldenbroek, I. Yamamoto, R. Lin, D. Greenstein, Translational control of the oogenic program by components of OMA ribonucleoprotein particles in Caenorhabditis elegans. Genetics 198, 1513-1533 (2014).

32. T. Tsukamoto, M. D. Gearhart, C. A. Spike, G. Huelgas-Morales, M. Mews, P. R. Boag, T. H. Beilharz, Greenstein, D., LIN-41 and OMA ribonucleoprotein complexes mediate a translational repression-to-activation switch controlling oocyte meiotic maturation and the oocyte-to-embryo transition in Caenorhabditis elegans. Genetics 206, 2007-2039 (2017).

33. A. Baudrimont, A. Penkner, A. Woglar, T. Machacek, C. Wegrostek, J. Gloggnitzer, A. Fridkin, F. Klein, Y. Gruenbaum, P. Pasierbek, V. Jantsch, Leptotene/zygotene chromosome movement via the SUN/KASH protein bridge in Caenorhabditis elegans. PLoS Genet 6, e1001219 (2010).

34. P. Thévenaz, U. E. Ruttimann, M. Unser, A pyramid approach to subpixel registration based on intensity. IEEE Trans Image Process 7, 27-41 (1998).

Acknowledgments: We thank G. W. Gant Luxton for encouraging us to pursue this project. We thank Abby Dernburg, Verena Jantsch, Sarit Smolikove, Daniel Starr, and Anne Villeneuve for providing antibodies. We thank Jack Powers, Caroline Spike, and Todd Starich for comments on 
the manuscript and encouragement. We thank Ana E. Garcia-Vedrenne for guidance on statistics.

We thank Nikon Instruments for use of the SoRa microscope. Some strains were provided by the Caenorhabditis Genetics Center. Funding: NIH (GM57173 and NS095109 to D. G.) and

Dystonia Medical Research Foundation's Barbara Oliver Memorial Dystonia Research Award to

G. H.-M. Author contributions: G. H.-M. and D. G. conceptualized the research and the

experimental strategy. G. H.-M. and T. T. defined OOC-5-associated proteins. G. H.-M. and G.

M. generated the C. elegans strains and conducted genetic analyses. G. H.-M and M. S.

conducted the microscopy and quantified the results. G. H.-M. and D. G. wrote the manuscript.

All authors contributed to data interpretation and commented on the manuscript. Competing

interests: The authors declare that they have no competing interests. Data and materials

availability: All data supporting the conclusions of this study are present in the paper or the

Supplemental Materials. C. elegans strains are available upon request.

\section{Supplementary Materials:}

Materials and Methods

Figures S1-S7

Tables S1-S5

Movies S1-S3

References (27-34) 

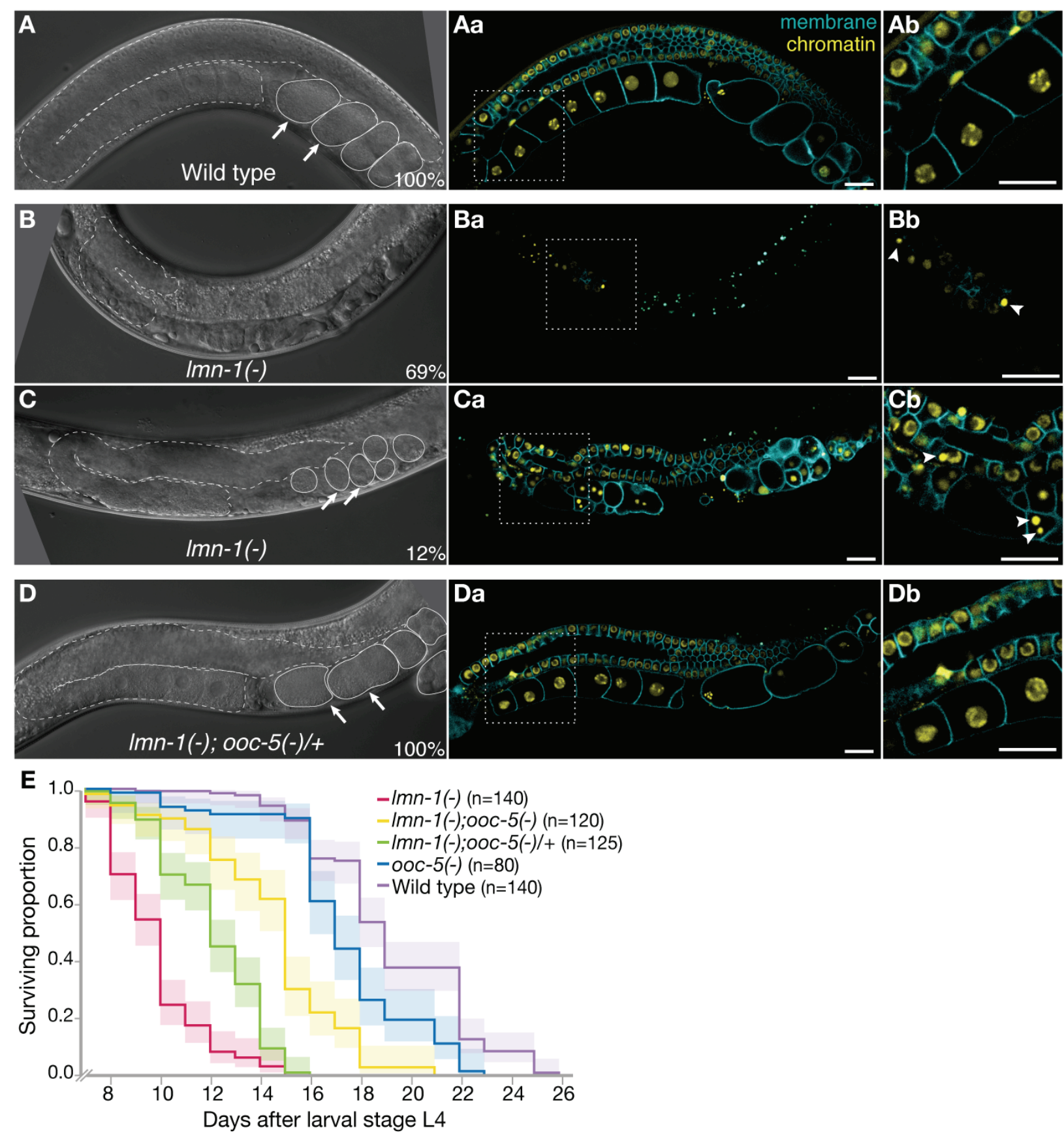

Fig. 1. Loss of ooc-5 rescues defects in lamin mutants. (A-D) DIC images of adult hermaphrodites. Arrows indicate embryos. (Aa-Da) Fluorescence micrographs of adult hermaphrodites. Cellular membranes of germ cells are visualized using a GFP::PH domain fusion and chromatin is visualized with a histone H2B::mCherry fusion. (Ab-Db) Insets are twice the size of the dotted-line region. Arrowheads indicate collapsing nuclei. Bar, $20 \mu \mathrm{m}$. (E) Kaplan-Meier 
bioRxiv preprint doi: https://doi.org/10.1101/2020.04.22.055988; this version posted April 23, 2020. The copyright holder for this preprint (which

was not certified by peer review) is the author/funder, who has granted bioRxiv a license to display the preprint in perpetuity. It is made available under aCC-BY-NC-ND 4.0 International license.

survival curves of the indicated genotypes. Three replicates were conducted, and confidence intervals are shadowed. 

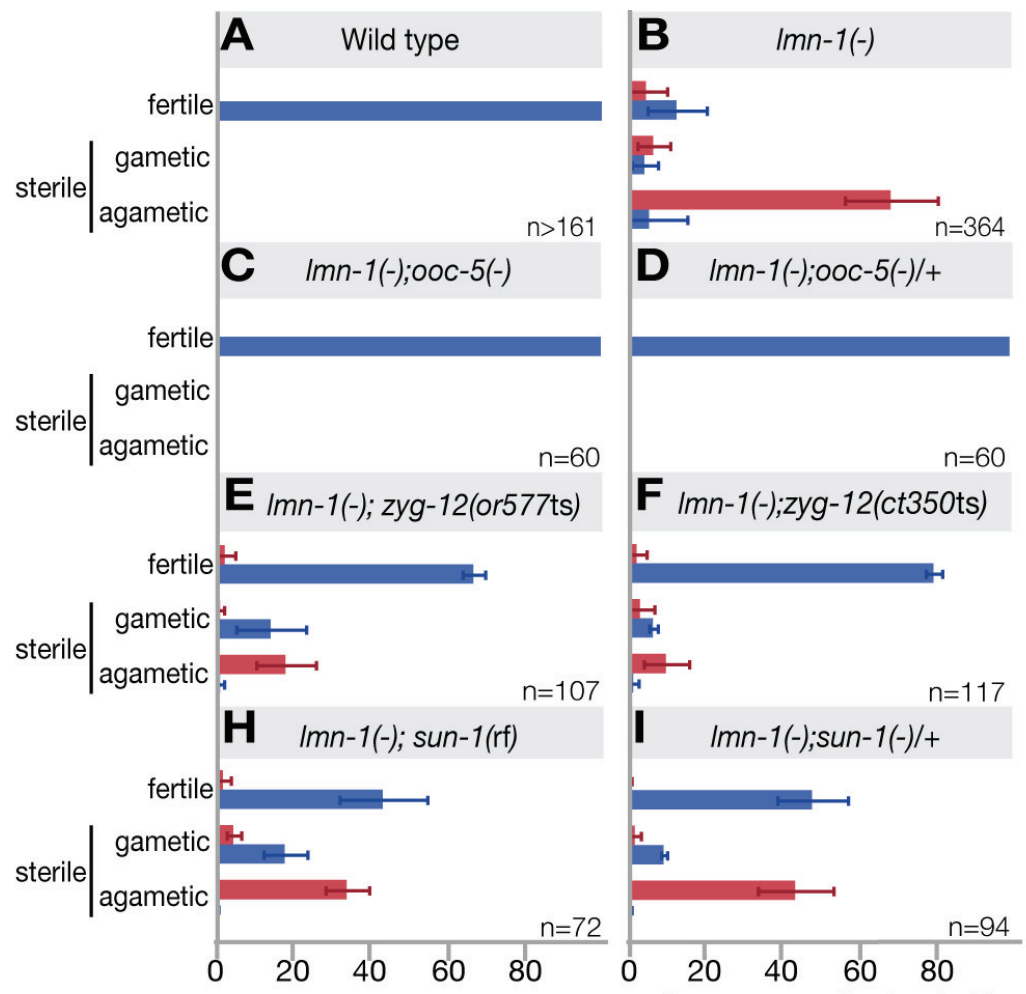

large gonad

(>300 germ cells)

small gonad

$(<300$ germ cells $)$
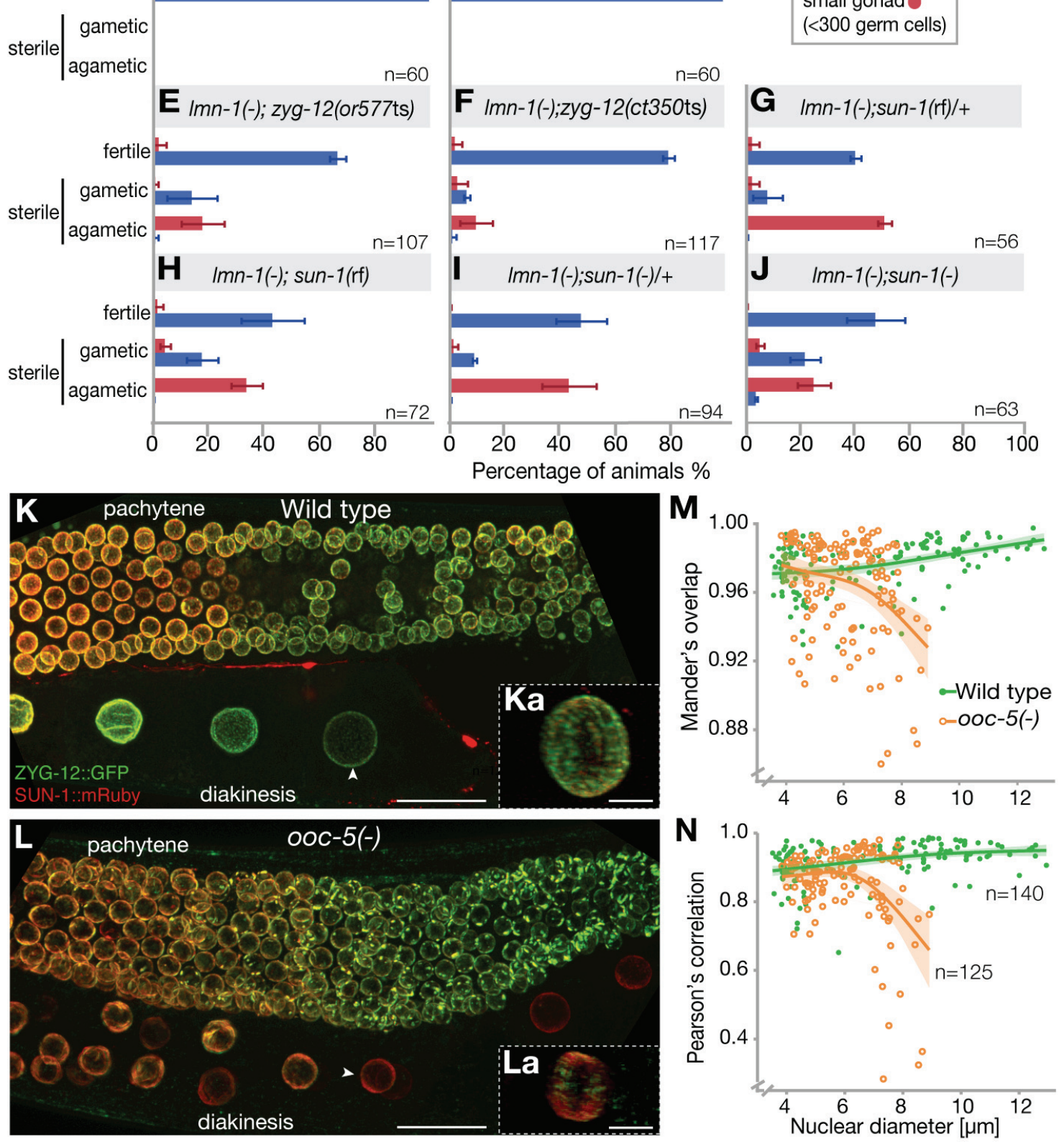

Fig. 2. ooc-5 regulates the LINC complex. (A-J) Hermaphrodites of the indicated genotypes grown at $20^{\circ} \mathrm{C}$, were classified as fertile ( $\geq 1$ embryo), sterile gametic (sperm/oocytes), or sterile agametic. Animals with more than 300 germ cells in a gonad arm were further classified as having 
large (blue) versus small gonads (red). ( $\mathbf{K}$ and $\mathbf{L})$ Maximum projections of Z-stack superresolution confocal images of ZYG-12::GFP and SUN-1::mRuby in the gonad. Bar, $20 \mu \mathrm{m}$. (Ka and La) 3D-reconstructions of the nuclei indicated with arrowheads. Bar, $5 \mu \mathrm{m}$. (M and $\mathbf{N}$ ) Colocalization of ZYG-12::GFP and SUN-1::mRuby in the NE. Bootstrap confidence regions are shadowed. 
A
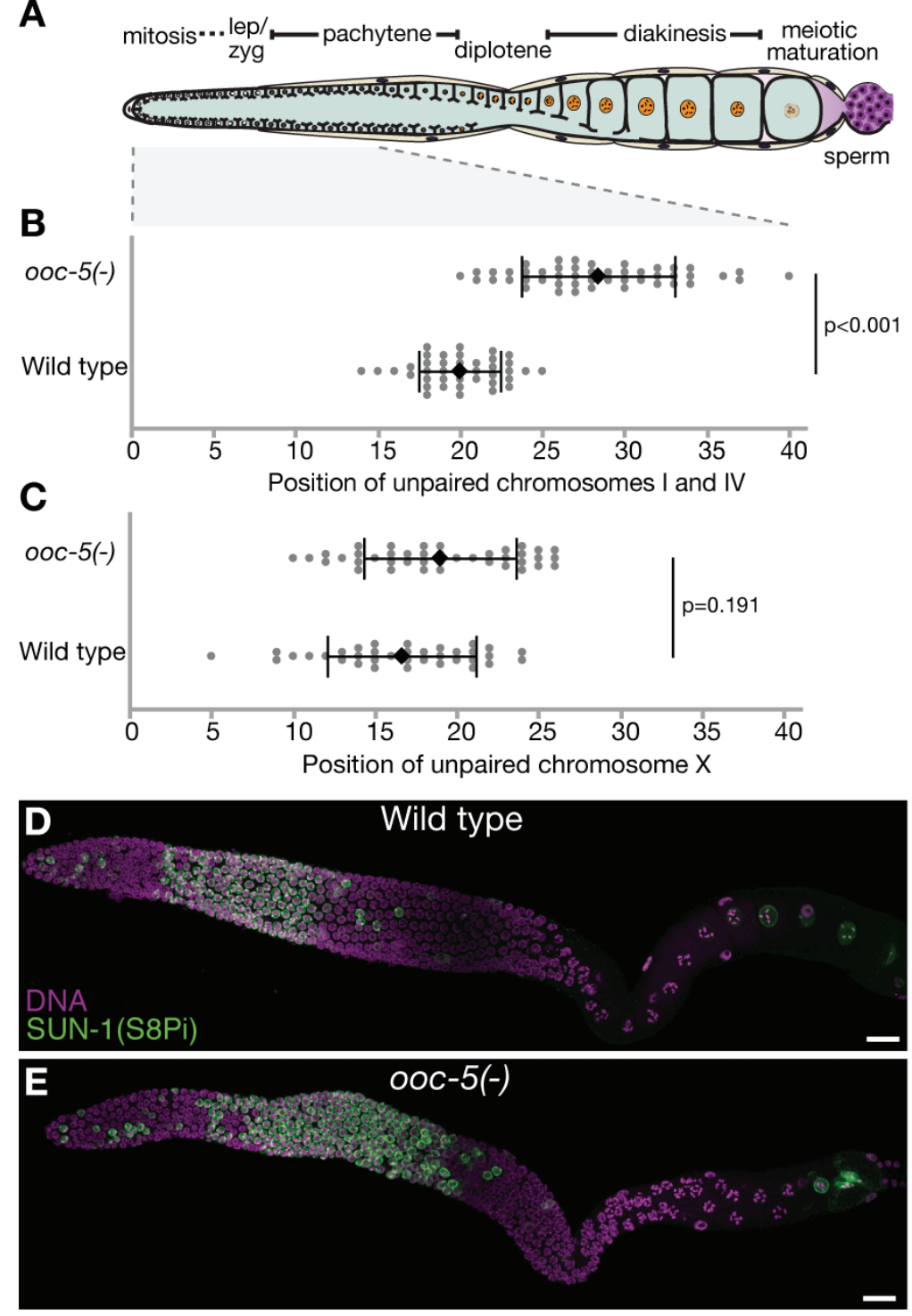

G
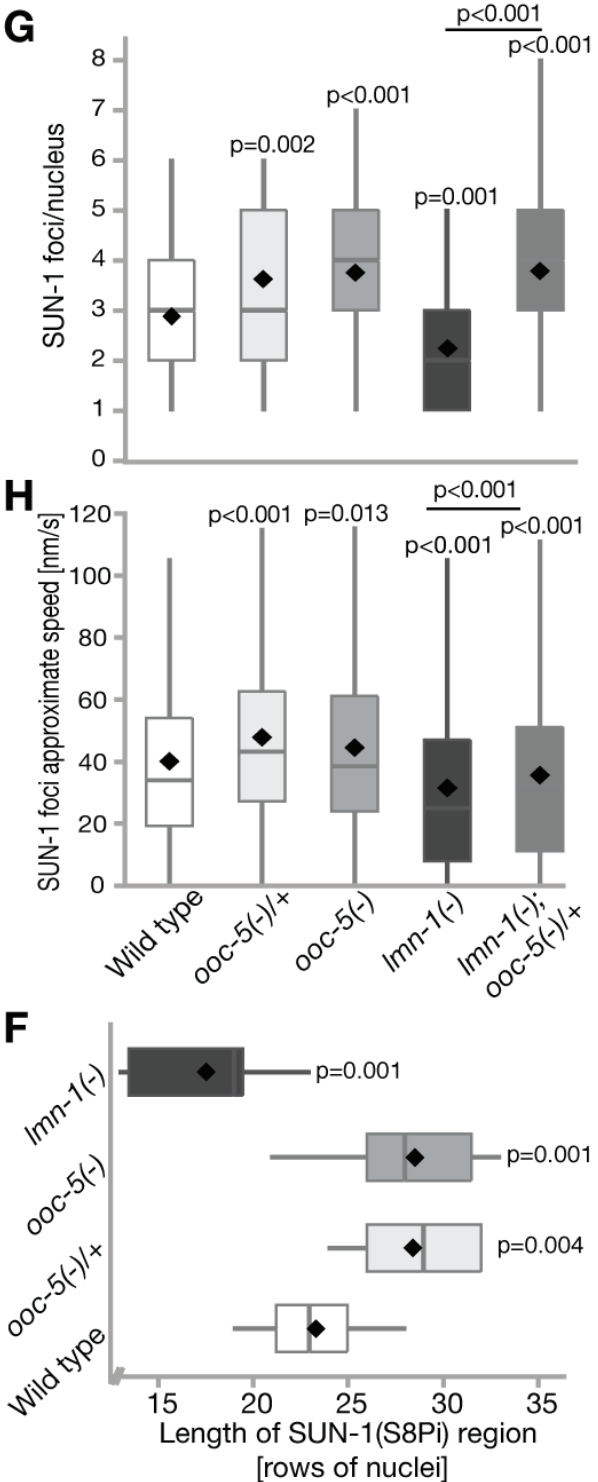

[rows of nuclei]

Fig. 3. LINC complex function during meiosis is compromised in ooc-5 mutants. (A) Diagram of the C. elegans adult hermaphrodite gonad. (B and C) Spatial distribution of unpaired chromosomes I and IV (B) and X chromosomes (C), visualized by immunostaining for ZIM-3 and HIM-8, respectively (see fig. S7). (D and E) Gonads immunostained for phosphorylated SUN-1 (S8Pi, green) and DNA (magenta). Bar, $20 \mu \mathrm{m}$. (F) Extent of the meiotic region containing phosphorylated SUN-1 (S8Pi). Diamonds indicate mean values. (G) Quantification of SUN-1 foci 
bioRxiv preprint doi: https://doi.org/10.1101/2020.04.22.055988; this version posted April 23, 2020. The copyright holder for this preprint (which

was not certified by peer review) is the author/funder, who has granted bioRxiv a license to display the preprint in perpetuity. It is made available under aCC-BY-NC-ND 4.0 International license.

per nucleus, and $(\mathbf{H})$ of their approximate speed of movement from time-lapse images (see mov.

S1-3). 

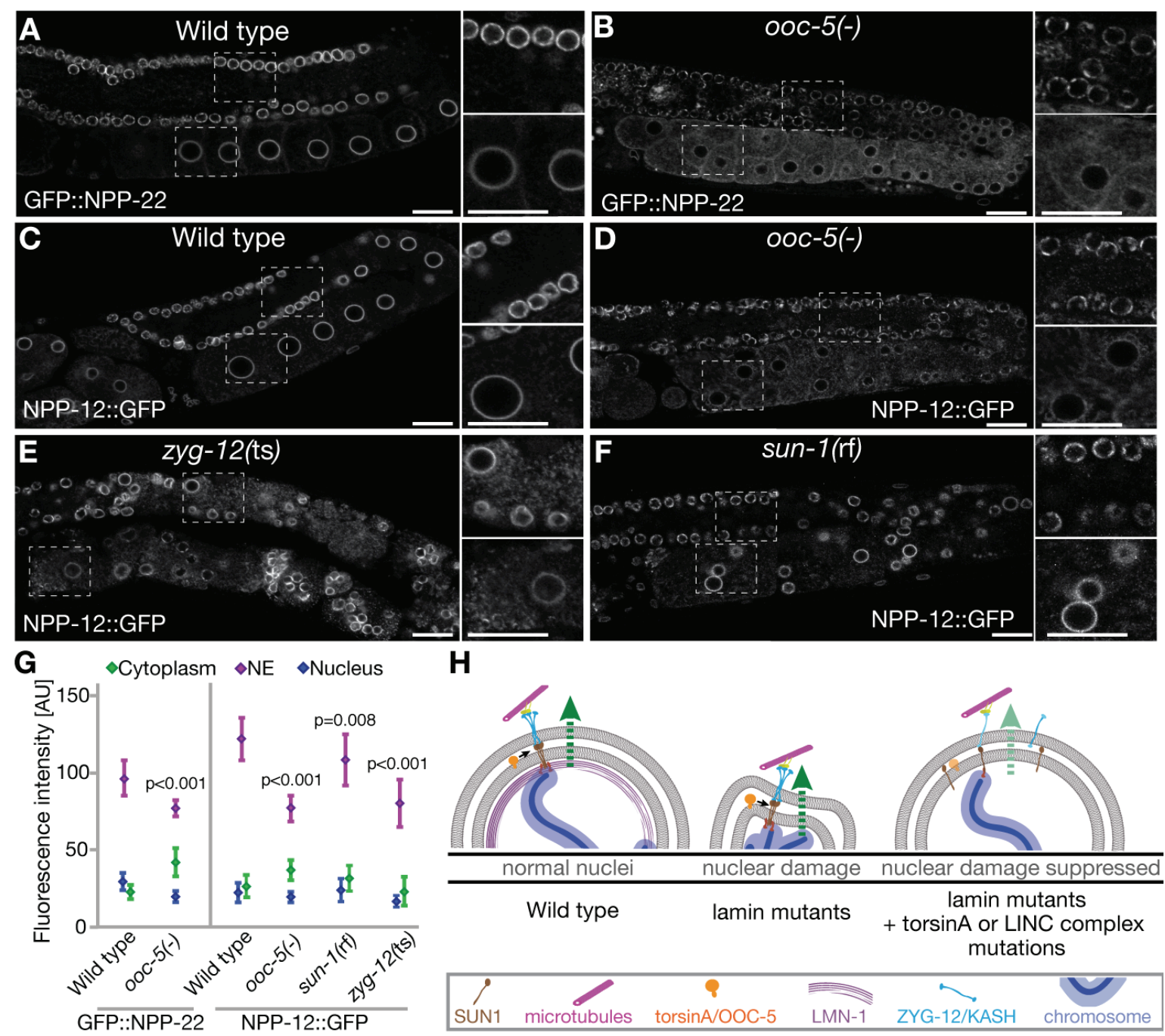

H

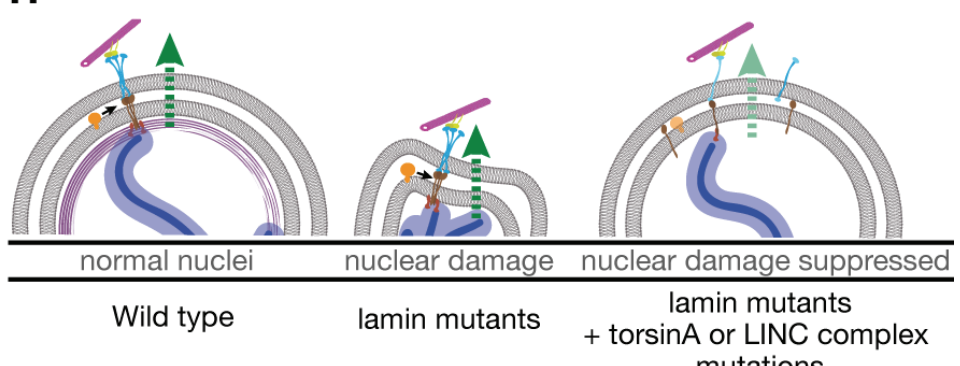

mutations

Fig. 4. OOC-5 and the LINC complex promote the localization of transmembrane Nups. (A-

F) Confocal images of the expression of GFP::NPP-22 or NPP-12::GFP in the gonad. Animals

were grown at $20^{\circ} \mathrm{C}$ except $z y g-12$ (ts) mutants, which were grown at $25^{\circ} \mathrm{C}$. Bars, $20 \mu \mathrm{m}$. (G)

Quantification of the fluorescence intensity of GFP::NPP-22 or NPP-12::GFP in subcellular compartments (mean \pm SD). (H) Model. OOC-5 promotes LINC complex function, damaging lmn1 mutant nuclei. Green arrows indicate force. 


\title{
Supplementary Materials for
}

\section{Decreased torsinA or LINC Complex Function Rescues a Laminopathy in \\ Caenorhabditis elegans}

Gabriela Huelgas-Morales ${ }^{1}$, Mark Sanders ${ }^{2}$, Gemechu Mekonnen ${ }^{1}$, Tatsuya Tsukamoto ${ }^{1}$, David Greenstein ${ }^{1 *}$.

\begin{abstract}
Affiliations: ${ }^{1}$ Department of Genetics, Cell Biology, and Development, University of Minnesota, Minneapolis, MN 55455 USA. ${ }^{2}$ University Imaging Center, University of Minnesota, Minneapolis, MN 55455 USA. *Correspondence to: David Greenstein. Email: green959@umn.edu.
\end{abstract}

This PDF file includes:

Materials and Methods

Supplemental References

figs. S1 to S7

tables S1 to S4

Captions for movies S1 to S3

Other Supplementary Materials for this manuscript include the following:

table S5

movies $\mathrm{S} 1$ to $\mathrm{S} 3$ 


\section{Materials and Methods}

\section{$\underline{\text { Strains }}$}

The genotypes of strains used are reported in the Supplement, table S4. Genes and mutations are described in WormBase (www.wormbase.org). The following mutations were used: LGI-lmn1(tm1502), npp-12(tn1780 [npp-12::gfp::tev::3xflag]), T09B4.2(tn1886); LGII-ooc-5(tn1757), ooc-5(it145) unc-4(e120), ooc-5(tn1709), ooc-5(tn1758[ooc-5::gfp::tev::3xflag]), zyg12(or577ts), zyg-12(ct350ts); LGIV-gei-18(tn1874); LGV-sun-1(jf18), sun-1(gk199), npp22(tn1794[gfp::tev::3xflag::npp-22]). The following rearrangements were used: tmC18[dpy5(tmIs1236)] I, mIn1[dpy-10(e128)mIs14] II, and tmC12[egl-9(tmIs1194)] V. The following transgenes and transgene insertions were used: ieSi21 [sun-1p::sun-1::mruby::sun-13'UTR+Cbr unc-119, itIs37[pie-1p::mCherry::H2B::pie-1 $\quad 3$ 'UTR + unc-119(+)] IV, ojIs9[zyg12(all)::gfp+unc-119(+)], and itIs38[pie-1p::GFP::PH(PLC1delta1)+unc-119(+)].

\section{Genome editing}

CRISPR-Cas9 genome editing used pRB1017 to express single guide RNA (sgRNA) under control of the C. elegans U6 promoter (27). To generate the sgRNA plasmids, we annealed oligonucleotides and ligated them to the pRB1017 vector, previously digested with BsaI. pDD162 was the source of Cas9, expressed under control of the eft-3 promoter (28).

We generated deletions in ooc-5 using a modification of the method of Paix et al. 2014 (29). Adult hermaphrodites of the N2 strain were injected with a mix containing the sgRNA plasmids at $25 \mu \mathrm{g} / \mathrm{ml}$ each, pDD162 at $50 \mu \mathrm{g} / \mathrm{ml}$, pmyo-2::tdTomato at $4 \mu \mathrm{g} / \mathrm{ml}, \mathrm{pCFJ} 104$ at $25 \mu \mathrm{g} / \mathrm{ml}$, and the oligonucleotide used as repair template at $500 \mathrm{nM}$. The oligonucleotides used as repair templates are listed in table S5. Transgenic animals from the F1 generation were singled out and 
pools of F2 were screened by the polymerase chain reaction (PCR). For the ooc-5 mutations F2 siblings from positive plates were crossed into the $m \operatorname{In} 1$ balancer.

We generated N-terminal or C-terminal translational fluorescent protein gene fusions using the self-excising-cassette method (30). The oligonucleotides to generate the repair templates are listed in table S5. All new alleles were verified by Sanger sequencing, and outcrossed to wild-type males at least three times.

\section{$\underline{\text { OOC-5 immunopurifications }}$}

OOC-5:: GFP::TEV::3xFLAG and interacting proteins were purified from the DG4377 strain. Purifications from the wild-type strain (N2) were run in parallel to use as control. The worms were grown at $15^{\circ} \mathrm{C}$ on peptone-enriched plates seeded with bacterial strain NA22. Embryos were isolated by alkaline hypochlorite treatment $(20 \%$ bleach and $0.5 \mathrm{~N} \mathrm{NaOH})$, washed in M9 buffer, and allowed to hatch overnight in the absence of food at $15^{\circ} \mathrm{C} .30,000 \mathrm{~L} 1$-stage animals were cultured on each $150 \times 15 \mathrm{~mm}$ petri dish on peptone-enriched medium with NA22 as a food source until they reached adulthood (60-70 petri dishes per strain).

To prepare the lysate, young adults were washed thoroughly with M9 and then frozen in droplets with liquid nitrogen. Then, the droplets were ground into fine powder, which was resuspended in an equal volume of lysis buffer, which was $75 \mathrm{mM}$ HEPES (pH 7.5), $1.5 \mathrm{mM}$ $\mathrm{MgCl}_{2}, 150 \mathrm{mM} \mathrm{KCl}, 15 \%$ glycerol and 1.5x protease inhibitors (cOmplete Mini EDTA-free, Roche). The crude extracts were sonicated for a total of $3 \mathrm{~min}$ at 30\% amplitude (Fisher Scientific, Model 500 Sonic Dismembrator) on ice. The soluble fraction was prepared by ultra-centrifugation of the crude extract at $135,000 \times \mathrm{g}$ for $30 \mathrm{~min}$ at $4^{\circ} \mathrm{C}$. The membrane fraction was prepared by resuspending the remaining pellet in lysis buffer plus 1\% Triton x-100 (Sigma-Aldrich), sonicating 
for $15 \mathrm{~s}$, and ultra-centrifuging at $135,000 \mathrm{x}$ g for $30 \mathrm{~min}$ at $4{ }^{\circ} \mathrm{C}$. The protein concentration of each fraction was determined by Bradford assay (Bio-Rad protein assay dye).

For the primary immunopurification of OOC-5::GFP::TEV:: 3xFLAG, Dynabeads Protein G (Life Technologies) were crosslinked to anti-FLAG M2 antibodies (Sigma-Aldrich, F1804), according to the manufacturer's instructions. The soluble (500 mg protein input) and membrane (170 $\mathrm{mg}$ protein input) fractions were incubated with these dynabeads for $1 \mathrm{~h}$ at $4{ }^{\circ} \mathrm{C}$ with rotational mixing. Then, the beads were washed $3 \mathrm{x}$ with IP wash buffer, which was 50 mM HEPES (pH 7.5), $1 \mathrm{mM} \mathrm{MgCl}_{2}, 300 \mathrm{mM} \mathrm{KCl}, 10 \%$ glycerol, $0.05 \%$ NP-40, 5 mM 2-mercaptoethanol, $5 \mathrm{mM}$ sodium citrate, $10 \mu \mathrm{M} \mathrm{ZnCl}$, and protease inhibitors (cOmplete Mini EDTA-free, Roche). Immunopurified proteins were eluted with $0.17 \mathrm{U} / \mathrm{ul}$ AcTEV protease (Invitrogen) in IP wash buffer for $16 \mathrm{~h}$ at $4^{\circ} \mathrm{C}$ with rotational mixing.

For the secondary immunopurification, the Dynabeads were crosslinked to a 1:1 mixture of anti-GFP 12A6 and anti-GFP 4C9 (Developmental Studies Hybridoma Bank, University of Iowa). The TEV-eluates were incubated with these Dynabeads for $1 \mathrm{~h}$ at $4^{\circ} \mathrm{C}$ with rotational mixing. Then, the beads were washed 6x with IP wash buffer. Immunopurified proteins were eluted with $100 \mathrm{mM}$ glycine ( $\mathrm{pH} 2.5)$ and neutralized immediately with an equal volume of $2 \mathrm{M}$ Tris- $\mathrm{Cl}(\mathrm{pH}$ 8.5). Small aliquots of protein fractions from every step of the purifications were separated on a 412\% Bis-Tris NuPAGE gel, transferred to Protran nitrocellulose membrane (Whatman), and it was incubated first with rabbit anti-GFP NB600-308 antibody (1:4,000) (Novus Biologicals) for 2 h, and then with peroxidase-conjugated donkey anti-rabbit antibody (1:7,000) (Jackson ImmunoResearch) (fig. S1F). Detection was performed using SuperSignal West Femto Maximum Sensitivity Substrate (Thermo Scientific). 
The glycine eluates containing immunopurified proteins were concentrated on a centrifugal evaporator (Jouan RC 10-10 Vacuum Concentrator) for 5 h, briefly separated on a 12\% Bis-Tris NuPAGE gel, and stained using the Colloidal Blue Staining Kit (Invitrogen) (fig. S1G). Lanes were subdivided into six slices and mass spectrometry was performed at the Taplin Biological Mass Spectrometry Facility (Harvard Medical School) using an LTQ ion-trap mass spectrometer. To account for abundant contaminant proteins that bound non-specifically to the immunopurification matrix, in addition to our control immunopurifications from the N2 strain, we utilized data from multiple purifications previously reported $(31,32)$.

\section{Lifespan analysis}

All the strains were maintained at $20^{\circ} \mathrm{C} .10$ mid-L4 hermaphrodites were transferred onto NGM plates freshly seeded with OP50-1. These animals were scored for viability every 1-3 days, either by movement or tapping them with a platinum wire. The wild-type animals were transferred to NGM plates from the same batch every other day for a week to avoid overcrowding by their progeny. The animals that crawled on the plate sides were censored. Kaplan-Meier survival curves were generated using the JMP Pro 14 software (SAS Institute Inc.). Wilcoxon tests were performed to individual pairs of survival curves.

\section{Immunostaining}

The gonads of day 1 adult hermaphrodites were dissected in Egg Salt buffer fixed in 1\% paraformaldehyde for $5 \mathrm{~min}$, and post fixed in cold methanol $\left(-20^{\circ} \mathrm{C}\right)$ for $1 \mathrm{~min}$. Then, the samples were blocked with $1 \mathrm{mg} / \mathrm{ml} \mathrm{BSA}$ in PBT overnight at $4^{\circ} \mathrm{C}$. Primary antibody incubation was 
performed in $\mathrm{PBT}+\mathrm{BSA}$ overnight at $4^{\circ} \mathrm{C}$, while secondary antibody incubation was performed in PBT+BSA for 1.5-2 $\mathrm{h}$ at room temperature.

Gonads were stained with the following antibodies provided by Abby Dernburg: goat antiSYP-1 (1:4000), rabbit anti-ZIM-3 (1:1000), guinea pig anti-HIM-8 (1:250), guinea pig anti-HTP3 (1:300). The guinea pig anti-SUN-1(S8Pi) (1:700) was provided by Verena Jantsch, and the rabbit anti-RAD-51 (1:30,000) was provided by Sarit Smolikov. Secondary antibodies were Cy3conjugated goat anti-mouse (1:500, Jackson ImmunoResearch), Cy3-conjugated donkey antichicken IgY (1:1000, Jackson ImmunoResearch), and DyLight 488-conjugated donkey antiguinea pig (1:800, Thermofisher Scientific). 4',6'-diamidino-2-phenylindole (DAPI) was used to detect DNA.

\section{Image acquisition and processing}

The images of day 1 adult hermaphrodites, anesthetized with $0.1 \%$ levamisole on $2 \%$ agarose pads, were acquired on a Nikon Ti2 inverted microscope equipped with a Plan Apo IR 60x and an HP Apo TIRF 100xH oil immersion objective lenses, NA 1.27 and 1.49 respectively. Illumination was provided by 405,488 and $561 \mathrm{~nm}$ lasers to the multi-point galvano and resonant scanners. The emission filters used were 450/50,525/50 and 595/50nm. The galvano scanner was used to acquire the images. For the images shown in the figures and movies, comparable acquisition parameters were used.

To examine the colocalization of SUN-1::mRuby and ZYG-12::GFP we used Spinning Disk Super Resolution by Optical Pixel Reassignment (SoRA). $0.30 \mu \mathrm{m}$ step z-series images were acquired in a Nikon Ti2 inverted microscope equipped with a SR HP plan apo lambda S 100X silicon oil immersion objective lens, NA 1.35. Illumination of the double-labeled samples was 
provided by 488 and $561 \mathrm{~nm}$ lasers fed through a single mode fiber to the CSU-W1 SoRA spinning disk confocal head with 50-micron pinhole. The emission filters used were 522/45 and 605/70nm, respectively. Images were acquired using a Photometrics Prime SCMOS camera with a pixel size of $0.13 \mu \mathrm{m}$ and the SoRA (1x mag) images were reconstructed. Images were deconvolved using the Nikon Elements 3D automatic deconvolution method, which utilized between 20 and 50 iterations.

The colocalization of SUN-1::mRuby and ZYG-12::GFP was quantified on images acquired with the SoRA for several nuclei from two specimens per strain, as well as on confocal images acquired with the Nikon Ti2 for several nuclei from 5 specimens per strain. The images acquired with the Nikon Ti2 were denoised and a threshold was set to include the fluorescence of SUN1::mRuby at the nuclear envelope. Mander's overlap and Pearson's coefficient were determined for each nucleus at the mid focal plane, and their nuclear diameters were measured. Imaging and processing were done in Nikon Elements versions 5.10 and 5.20.

Nikon Elements GA3 was used to quantify the intensity of the fluorescence of NPP-12::GFP and GFP::NPP-22 from confocal images of day 1 adult hermaphrodite gonads. The $488 \mathrm{~nm}$ channel was processed as follows: the background was corrected with Rolling Ball (14 $\mu \mathrm{m})$ function, one pass of denoising was performed, and the regional maxima and Best FocusPlane were detected. Finally, a threshold was set for identification of the features of interest. Oocyte nuclei were filtered by circularity and fluorescence intensity values were measured (mean, min and max) at the NE, inside the NE (nucleoplasm) and at the cytoplasm (rectangular region between the edge of the NE and the cell membrane) for 10 oocytes per animal, from at least 2 animals per strain. The brightness and contrast of the images shown in Figure 4 were adjusted for presentation identically across all the strains. 
Time lapse acquisition and processing

The movement of SUN-1 foci was documented as previously reported with the following modifications (33). Hermaphrodites were selected as mid-L4 larvae and incubated at $20^{\circ} \mathrm{C}$. About $24 \mathrm{~h}$ later, young adults were anesthetized in $10 \mathrm{mM}$ levamisole and mounted on $2 \%$ agarose pads. $0.25 \mu \mathrm{m}$ step z-series were acquired with the Apo IR 60x WI, 1.27 NA objective approximately every $15 \mathrm{~s}$ for 6-7 min per animal. Maximum intensity projections were made with ImageJ (FIJI), the images were converted to grayscale and their Lookup Tables (LUTs) inverted. Individual SUN1 foci were followed using StackReg (34) and manual tracking plug-ins. The speed was determined for 84 to 390 individual foci per animal, and the number of animals assessed was equal or greater than 3 per strain.

\section{$\underline{\text { Statistics }}$}

The statistical analyses described below were performed using JMP Pro v14 software (Statistical Discovery, SAS). For the suppression of sterility phenotype data, a contingency analysis of outcome by genotype was performed (see fig. S2). Standard ChiSquare tests were conducted to compare the data from each mutant to those of their respective control (e.g. lmn-1(-

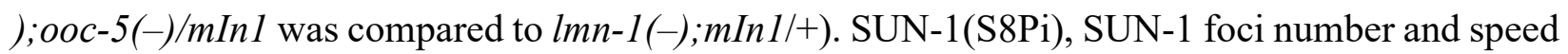
of movement, and the position of unpaired chromosomes data were tested for equality of group variance. Since they all had unequal variances, non-parametric comparisons were made for each pair using the Wilcoxon method.

The comparison of fluorescence intensity of NPP-12::GFP or GFP::NPP-22 in different subcellular compartments between genetic backgrounds was performed using Prism 8 (GraphPad 
software, LLC.). A 2way ANOVA showed that the effect of the different genetic backgrounds was significant, $\mathrm{P}<0.0001$. Bonferroni multiple comparisons tests were performed using the fluorescent intensity of the nucleoporins in the otherwise wild-type background as controls.

Figure $4 \mathrm{G}$ shows the p-values for these comparisons in the NE.

\section{$\underline{\text { Supplemental References }}$}

27. J. A. Arribere, R. T. Bell, B. X. Fu, K. L. Artiles, P. S. Hartman, A. Z. Fire, Efficient markerfree recovery of custom genetic modifications with CRISPR/Cas9 in Caenorhabditis elegans. Genetics 198, 837-846 (2014).

28. D. J. Dickinson, J. D. Ward, D. J. Reiner, B. Goldstein, Engineering the Caenorhabditis elegans genome using Cas9-triggered homologous recombination. Nat Methods 10, 1028-1034 (2013).

29. A. Paix, Y. Wang, H. E. Smith, C. Y. Lee, D. Calidas, T. Lu, J. Smith, H. Schmidt, M. W. Krause, G. Seydoux, Scalable and versatile genome editing using linear DNAs with microhomology to Cas9 Sites in Caenorhabditis elegans. Genetics 198, 1347-1356 (2014).

30. D. J. Dickinson, A. M. Pani, J. K. Heppert, C. D. Higgins, B. Goldstein, Streamlined genome engineering with a self-excising drug selection cassette. Genetics 200, 1035-1049 (2015).

31. C. A. Spike, D. Coetzee, Y. Nishi, T. Guven-Ozkan, M. Oldenbroek, I. Yamamoto, R. Lin, D. Greenstein, Translational control of the oogenic program by components of OMA ribonucleoprotein particles in Caenorhabditis elegans. Genetics 198, 1513-1533 (2014). 
32. T. Tsukamoto, M. D. Gearhart, C. A. Spike, G. Huelgas-Morales, M. Mews, P. R. Boag, T.

H. Beilharz, Greenstein, D., LIN-41 and OMA ribonucleoprotein complexes mediate a translational repression-to-activation switch controlling oocyte meiotic maturation and the oocyte-to-embryo transition in Caenorhabditis elegans. Genetics 206, 2007-2039 (2017).

33. A. Baudrimont, A. Penkner, A. Woglar, T. Machacek, C. Wegrostek, J. Gloggnitzer, A.

Fridkin, F. Klein, Y. Gruenbaum, P. Pasierbek, V. Jantsch, Leptotene/zygotene chromosome movement via the SUN/KASH protein bridge in Caenorhabditis elegans. PLoS Genet 6, e1001219 (2010).

34. P. Thévenaz, U. E. Ruttimann, M. Unser, A pyramid approach to subpixel registration based on intensity. IEEE Trans Image Process 7, 27-41 (1998). 
bioRxiv preprint doi: https://doi.org/10.1101/2020.04.22.055988; this version posted April 23, 2020. The copyright holder for this preprint (which was not certified by peer review) is the author/funder, who has granted bioRxiv a license to display the preprint in perpetuity. It is made available under aCC-BY-NC-ND 4.0 International license.

A

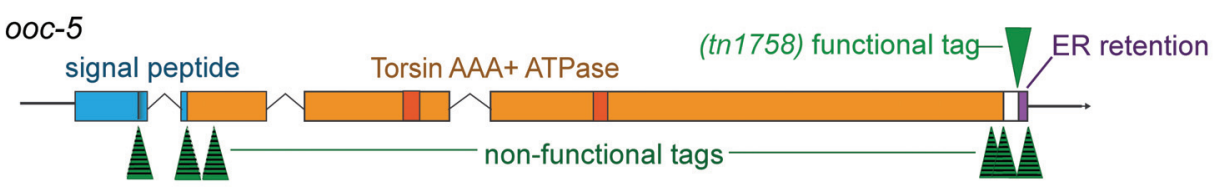

ooc-5(tn1757)

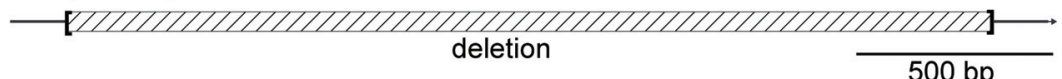
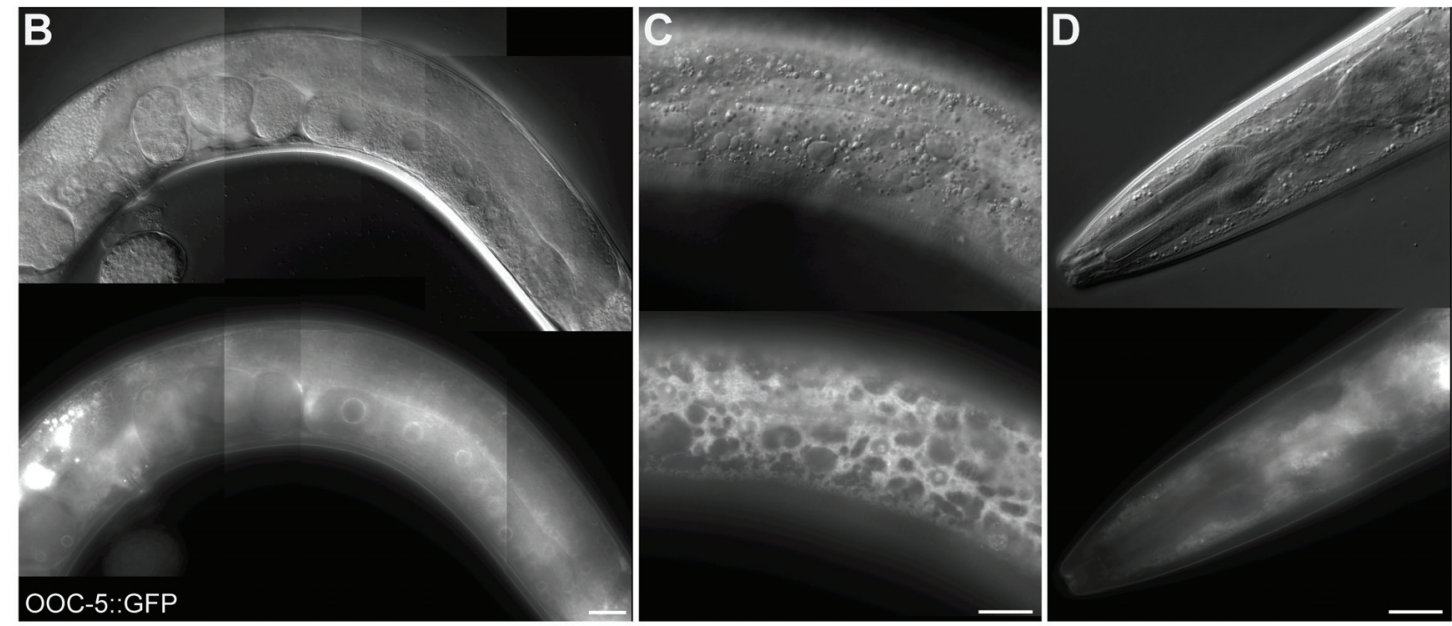

E

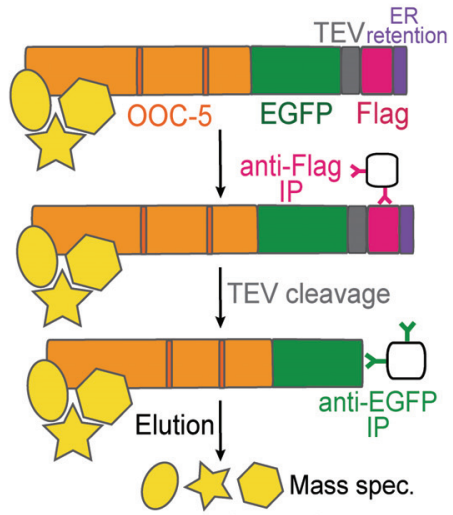

NPP-12, NPP-22, SUN-1, SAMP-1,

OOC-3, CNX-1, LMN-1 and others

G

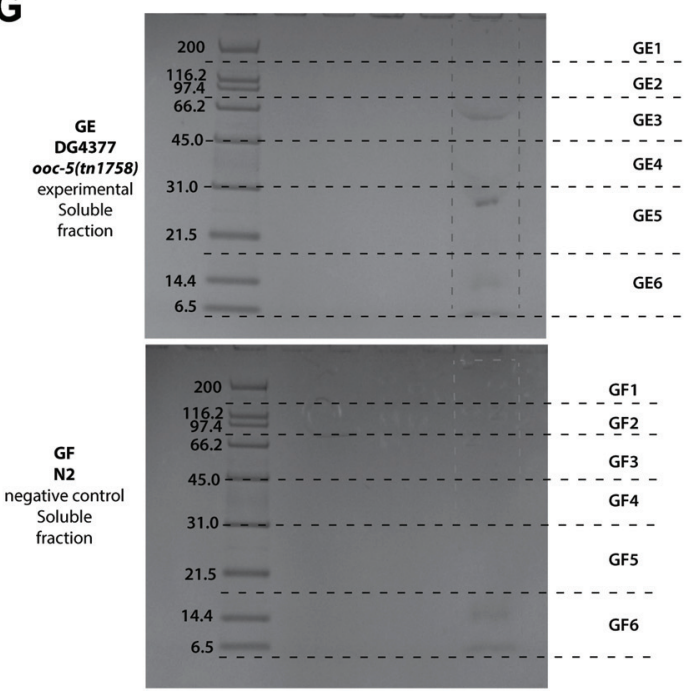

$\mathbf{F}$

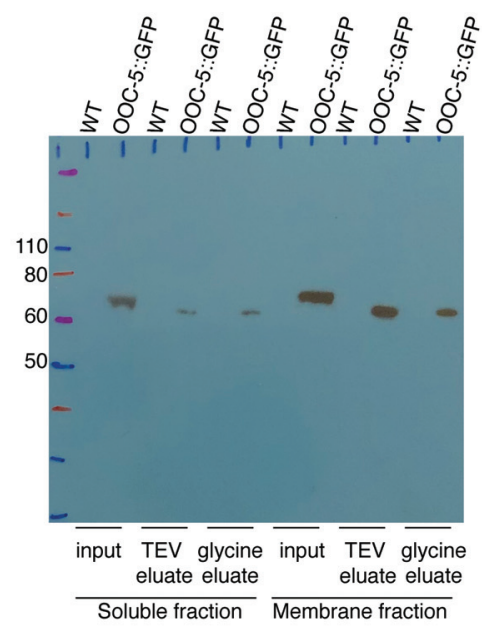


fig. S1. OOC-5/torsinA tandem affinity purification. (A-B) Representation of ooc-5 locus. Above: The affinity tag was inserted in 7 different positions by genome editing, but only the insertion right before the endogenous ER retention motif (HFDDEL) exhibited functionality. The product of the new allele ooc-5(tn1758) is herein referred to as OOC-5::GFP. Although the OOC-5::GFP strain was viable and fertile at $15^{\circ} \mathrm{C}$, it was temperature-sensitive (table S2). Thus, purifications were conducted only using animals grown at $15^{\circ} \mathrm{C}$. Below: Representation of the ooc-5(tn1757) null allele generated by genome editing. (B-D) DIC and GFP composite images of the expression of OOC-5::GFP in the gonad (B), hypodermis (C), and head (D) of a young adult hermaphrodite grown at $15^{\circ} \mathrm{C}$. Bars, $20 \mu \mathrm{m}$. (E) Overview of purification methods used to characterize OOC-5-associated proteins. GFP (green), tobacco etch virus (TEV) protease cleavage site (gray), and 3x Flag-tag (pink). (F) Western blot of the steps involved in OOC5::GFP tandem affinity purification, from both the soluble and the membrane fractions. Note, the bands decreased in size slightly after TEV protease cleavage as expected, from approximately 73 to $68 \mathrm{KDa}$. (G) Coomassie staining of the OOC-5::GFP (GE and GG) and control (N2) (GF and $\mathrm{GH}$ ) immunopurifications, for soluble and membrane fractions of the protein extracts. The lanes were cut into six slices each and processed by mass spectrometry. 


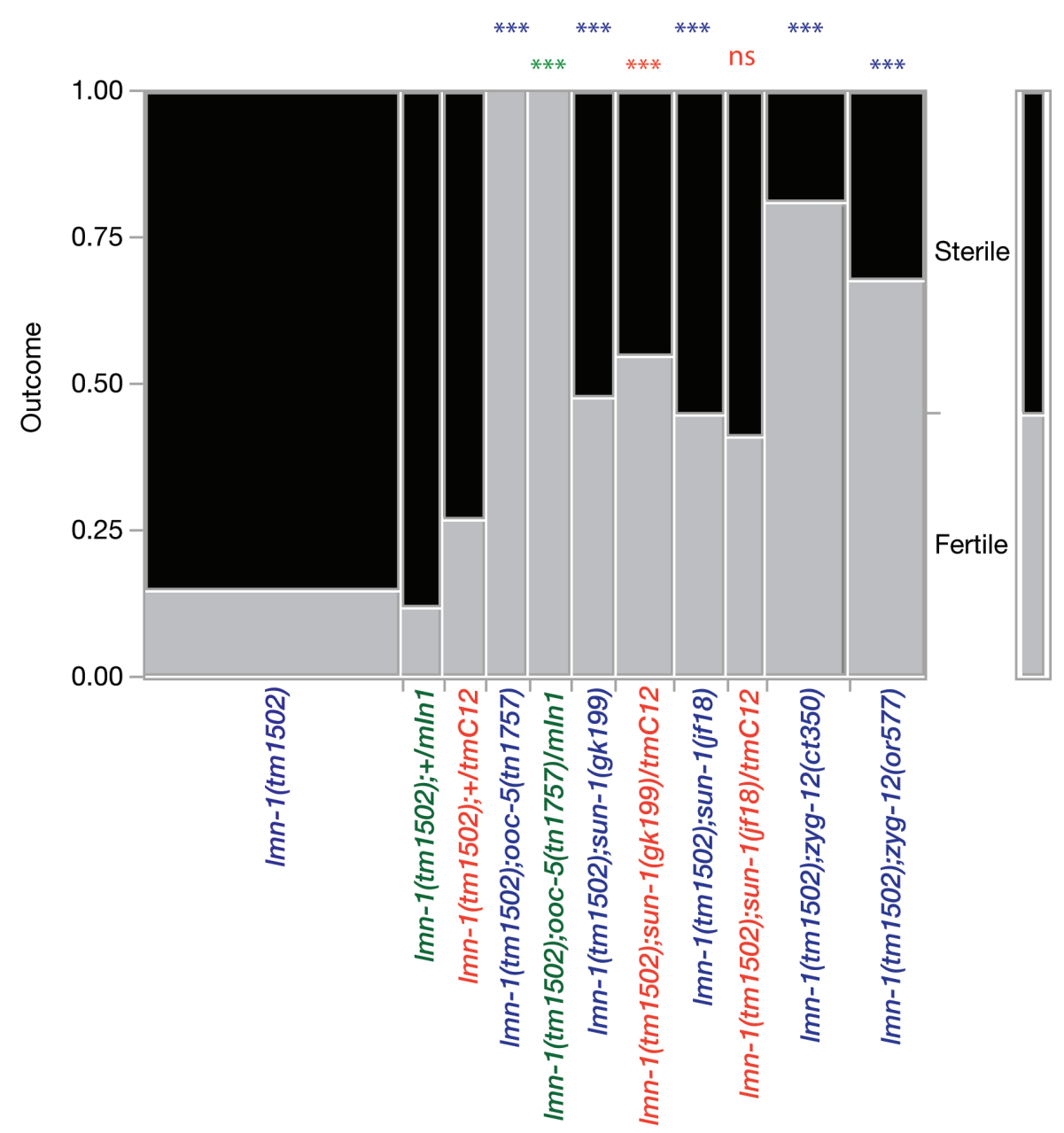

fig. S2. Mutations in ooc-5 and in the LINC complex components rescue the sterility of Imn-

1 mutants. The observations of the phenotypes depicted in Figure 2, panels A-J were classified into fertile and sterile to perform statistical tests. This table allowed for the contingency analysis of the data. Chi square tests were performed by comparing each strain to its specific control accounting for balancers in the genetic backgrounds analyzed (strains in blue were compared to the corresponding control strain in blue, same with green and orange color coded strains), *** means $\mathrm{p}<0.001$ and $\mathrm{ns} \mathrm{p}=0.1$. In addition to the ooc-5(tn1757) allele, we observed that the sterility of $l m n-1(-)$ was also suppressed by ooc-5(tn 1709) and ooc-5(it145). 

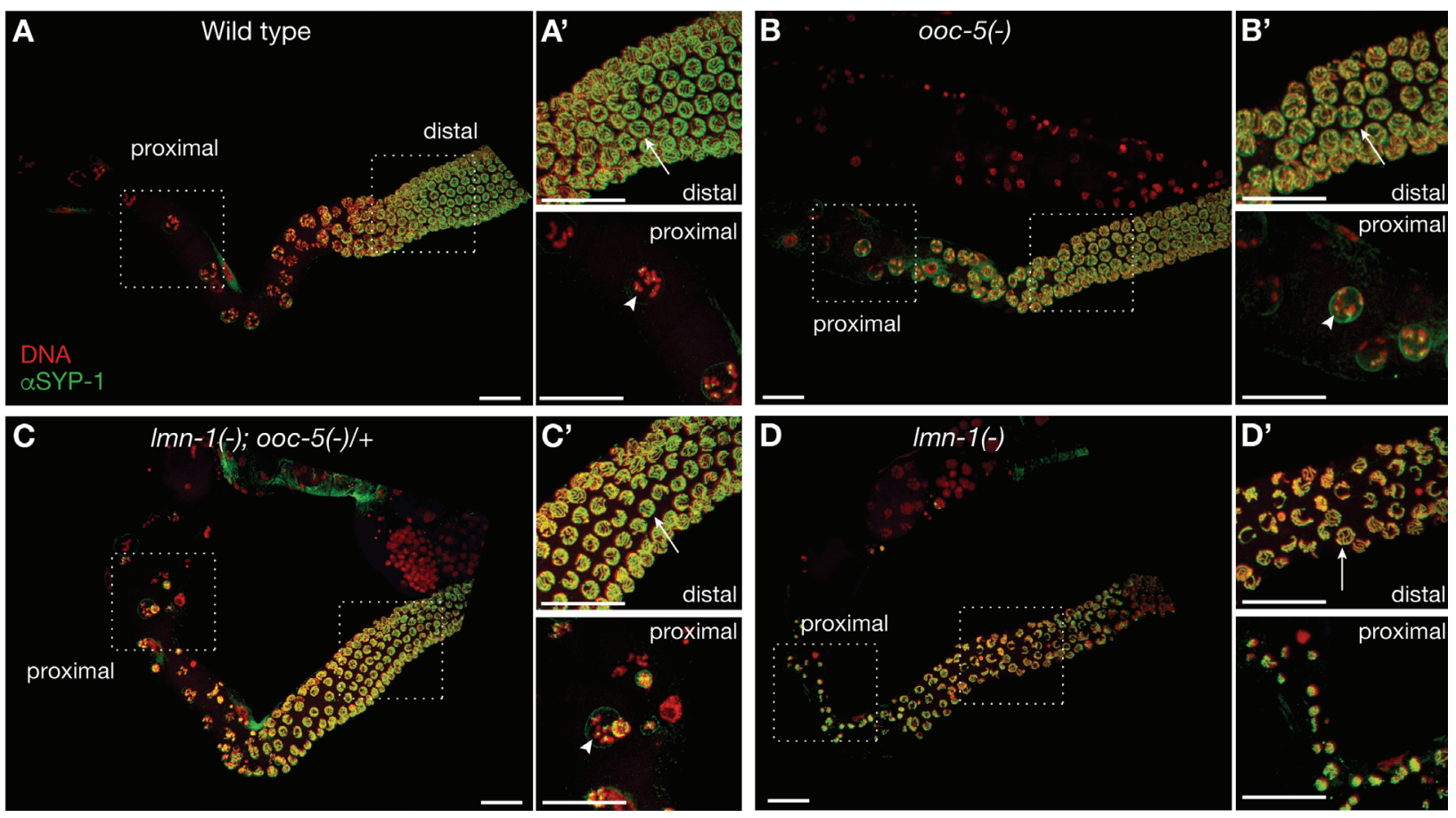

\section{fig. S3. Imn-1 mutants assemble a synaptonemal complex, but their nuclei collapse after}

pachytene. Day 1 adult hermaphrodites of the indicated genotypes were dissected, fixed and immunostained for SYP-1(green) and DNA (DAPI, red). (D) The $l m n-1$ mutant shown here is an “escaper", which produced dead embryos (see main text). (A'-D') Insets are two-fold magnified relative to the main images. Note, synaptonemal complexes are visible in pachytene nuclei (arrows) of $l m n-1(-)$ mutants (distal D'), but must nuclei collapse soon after (proximal D'). In contrast, diakinetic chromosomes (arrowheads) are present in $l m n-1(-)$; ooc-5(-)/+ animals (proximal C'). Bars, $20 \mu \mathrm{m}$. 

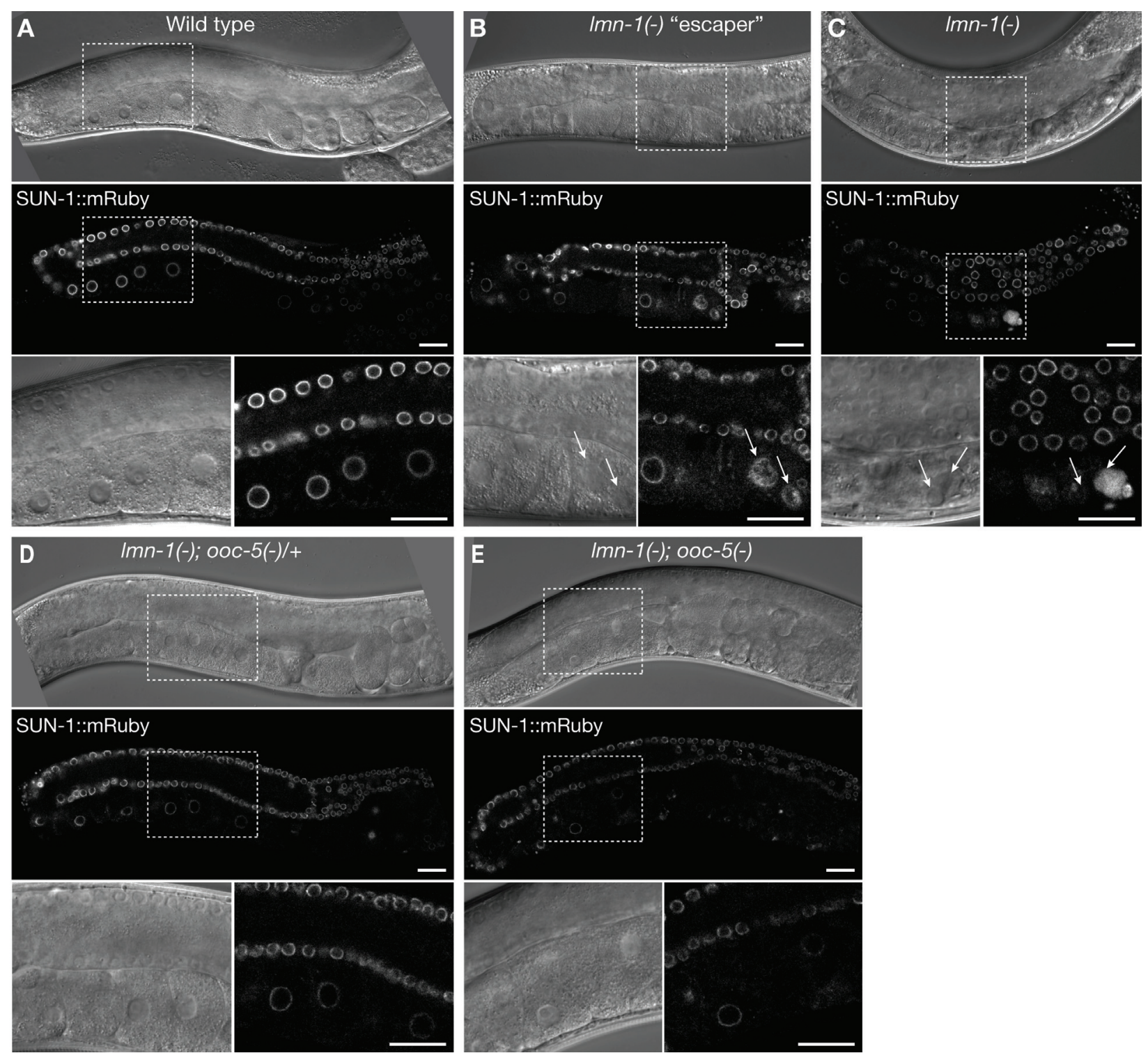

fig. S4. Nuclear damage in $l m n-1$ mutants is rescued by reducing ooc-5 function. (A-E)

Confocal and DIC images of the expression of SUN-1::mRuby in the gonad of animals of the indicated genotypes. Animals were grown at $20^{\circ} \mathrm{C}$. Insets are two-fold magnified relative to the main images. Arrows in the insets of $l m n-1(-)$ mutants (B and C) indicate damaged nuclei with SUN-1::mRuby diffuse localization. Bars, $20 \mu \mathrm{m}$. 


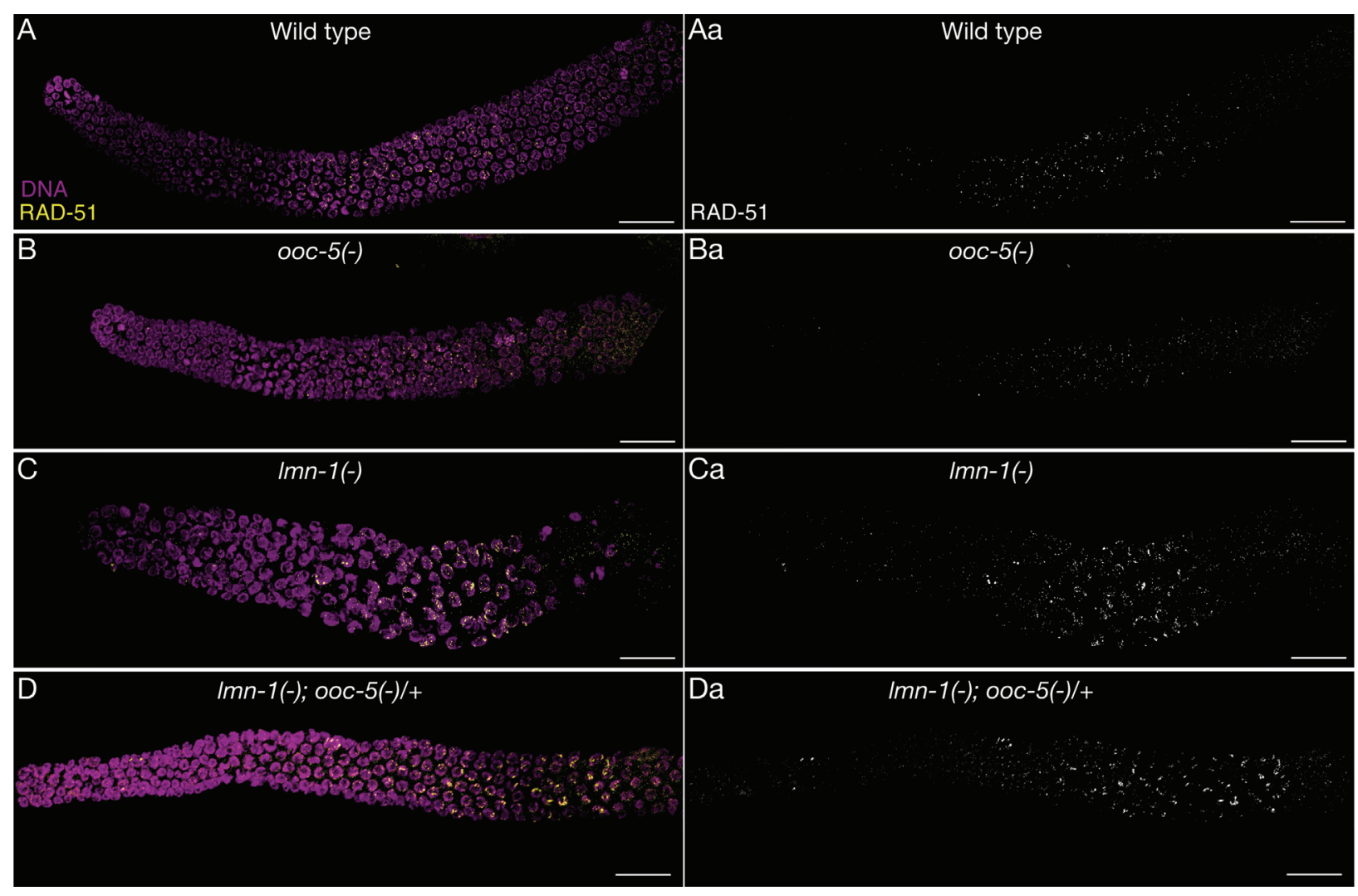

fig. S5. DNA damage in $l m n-1$ mutants is not rescued by decreasing ooc-5 function. (A-D)

Day 1 adult hermaphrodites of the indicated genotypes were dissected, fixed and immunostained for RAD-51 (yellow) and DNA (DAPI, magenta). RAD-51 is recruited to sites of DNA double strand breaks to promote successful crossover in normal meiotic cells. (Aa-Da) Color channels were split in A-B to show grayscale images for the RAD-51 staining only. Note, RAD-51 foci increased in size and number in both $l m n-1(-)(\mathrm{C})$ and $l m n-1(-) ;$ ooc-5(-)/+ (D) mutant strains, suggesting increased DNA damage in these genetic backgrounds. (D) The lmn-1 mutant shown here is an "escaper," which produced dead embryos (see main text). Bars, $20 \mu \mathrm{m}$. 


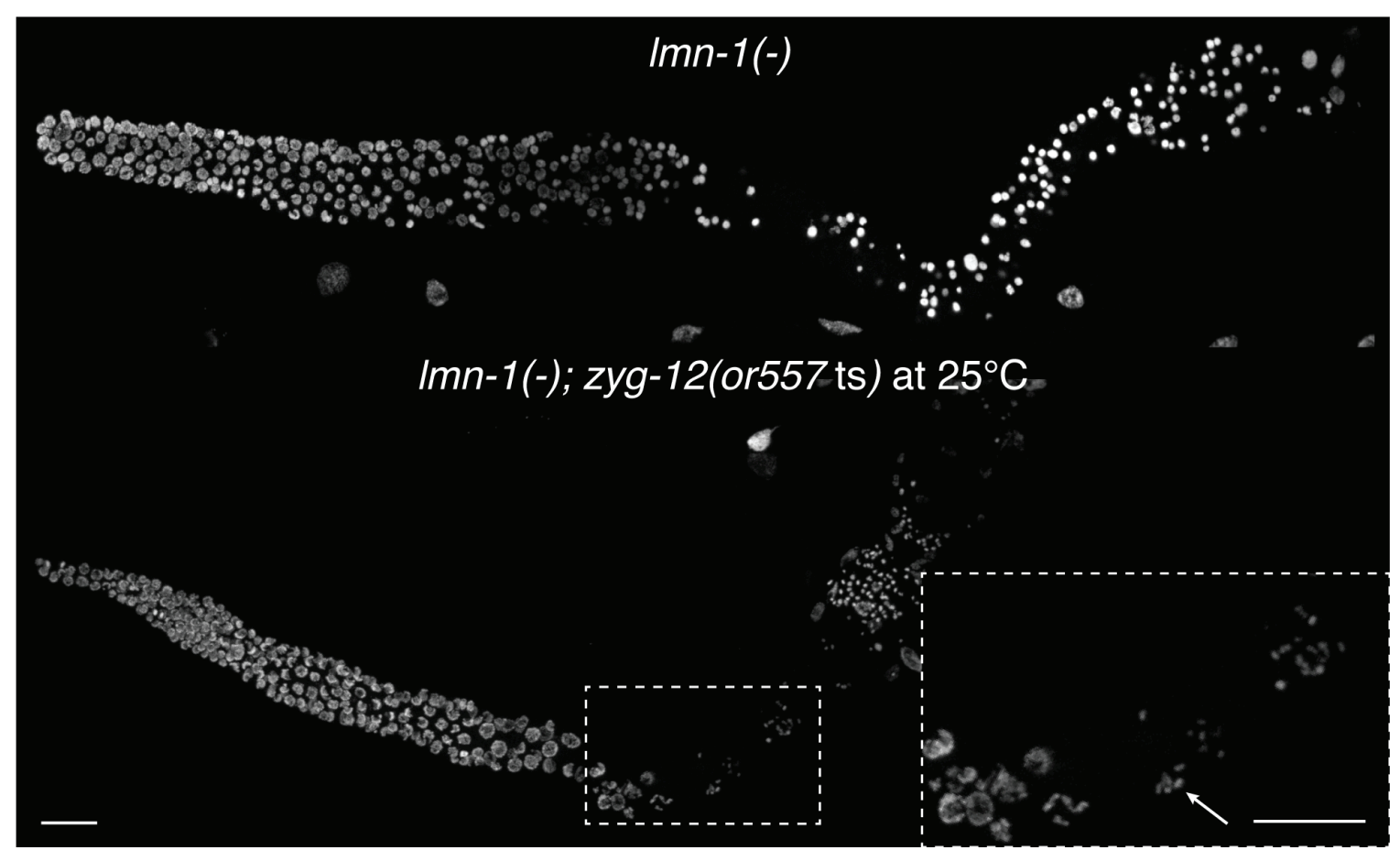

\section{fig. S6. Nuclear damage in Imn-1 mutants is ameliorated by decreasing LINC complex}

function. Animals were grown at $20^{\circ} \mathrm{C}$ until the L4 stage, then $\operatorname{lmn}-1(-)$; zyg-12(or557ts) double mutants were upshifted to $25^{\circ} \mathrm{C}$. $\operatorname{lmn}-1(-)$ single mutants were kept and analyzed at $20^{\circ} \mathrm{C}$ because they become sickly when grown at $25^{\circ} \mathrm{C}$ (unpublished results). Day 1 adult hermaphrodites were dissected, fixed and stained for DNA with DAPI. Note, diakinesis figures are observed in $l m n-1(-)$; zyg-12(or557ts) double mutants (arrow) but not $l m n-1(-)$ single mutants. Bars, $20 \mu \mathrm{m}$. 

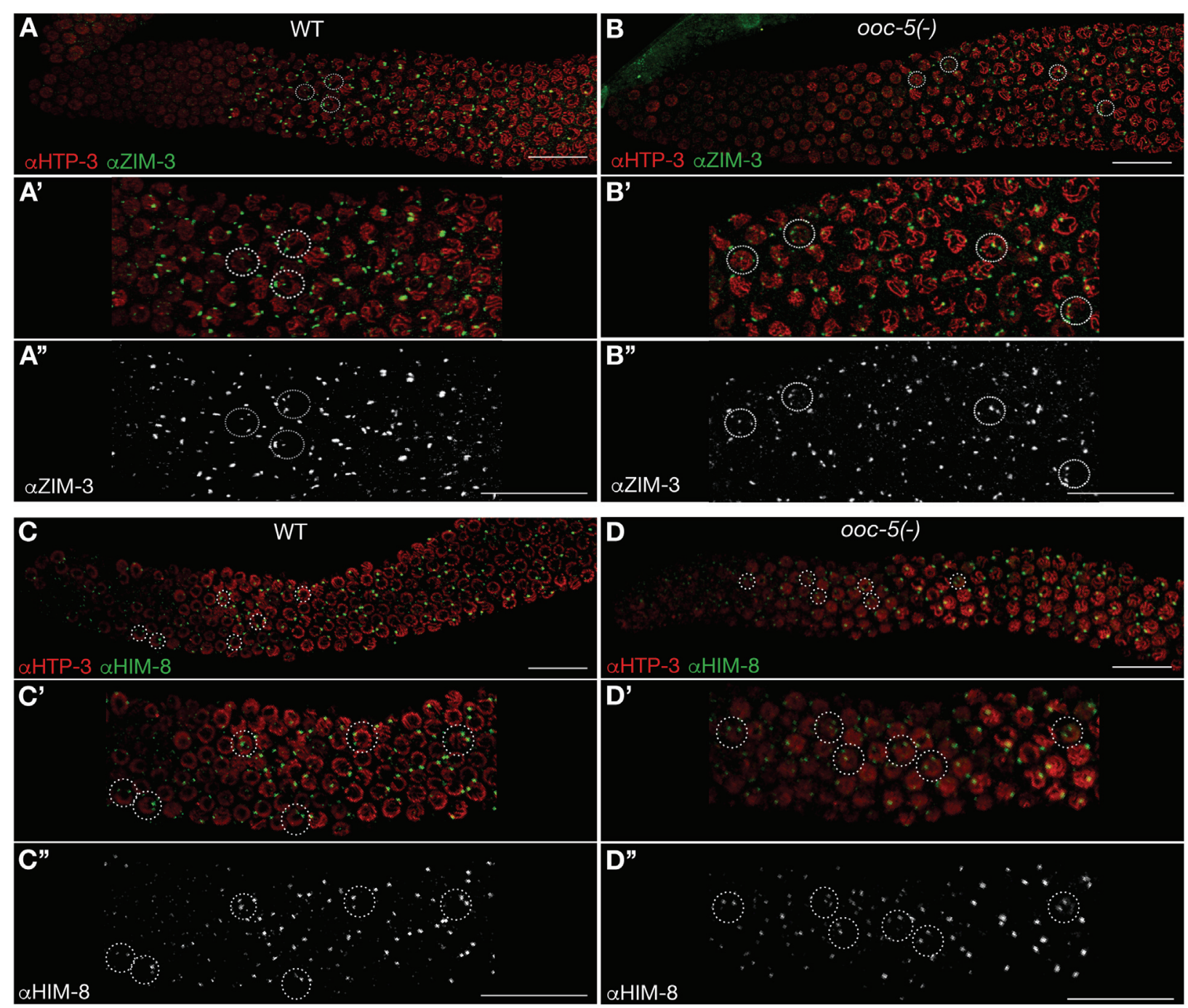

fig. S7. Autosome pairing is delayed in ooc-5 mutants. Day 1 adult hermaphrodites of the indicated genotypes were dissected, fixed and stained for the axial element protein HTP-3, and either ZIM-3 (A and B) or HIM-8 (C and D) to determine the position of unpaired chromosomes I and IV, and X chromosomes, respectively. Nuclei with unpaired meiotic chromosomes are shown inside the dotted circles. (A'-D' and A''-D'') Insets are two-fold magnified relative to the main images. Bars, $20 \mu \mathrm{m}$. 
Soluble fraction

(500 mg protein input)
Membrane fraction

(170 mg protein input)

\begin{tabular}{llll}
\hline OOC-5::GFP & Control & OOC-5::GFP & Control \\
\hline
\end{tabular}

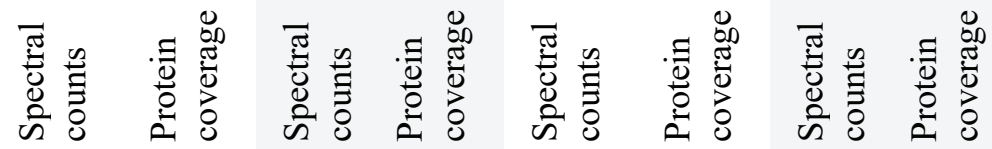

Human

homolog

\begin{tabular}{|c|c|c|c|c|c|c|c|c|c|}
\hline OOC-5 & 105 & $56 \%$ & 0 & $0 \%$ & 106 & $62 \%$ & 0 & $0 \%$ & TOR1A \\
\hline GFP & 72 & $74 \%$ & 0 & $0 \%$ & 69 & $82 \%$ & 0 & $0 \%$ & N/A \\
\hline RIBO-1 & 30 & $48 \%$ & 0 & $0 \%$ & 35 & $49 \%$ & 0 & $0 \%$ & Ribophorin-1 \\
\hline NPP-12 & 19 & $15 \%$ & 0 & $0 \%$ & 16 & $12 \%$ & 0 & $0 \%$ & gp210 \\
\hline SAMP-1 & 17 & $19 \%$ & 0 & $0 \%$ & 7 & $10 \%$ & 0 & $0 \%$ & TMEM201 \\
\hline OOC-3 & 6 & $12 \%$ & 0 & $0 \%$ & 5 & $15 \%$ & 0 & $0 \%$ & N/A \\
\hline UGT-49 & 7 & $18 \%$ & 0 & $0 \%$ & 4 & $10 \%$ & 0 & $0 \%$ & UGT3A2 \\
\hline CNX-1 & 4 & $8 \%$ & 0 & $0 \%$ & 1 & $2 \%$ & 0 & $0 \%$ & Calnexin \\
\hline LMN-1 & 20 & $43 \%$ & 0 & $0 \%$ & 18 & $42 \%$ & 0 & $0 \%$ & Lamin \\
\hline ATLN-1 & 4 & $12 \%$ & 0 & $0 \%$ & 15 & $28 \%$ & 0 & $0 \%$ & Atlastin \\
\hline T13H5.8 & 14 & $18 \%$ & 0 & $0 \%$ & 0 & $0 \%$ & 0 & $0 \%$ & CLPTM1 \\
\hline PDI-1 & 6 & $15 \%$ & 0 & $0 \%$ & 8 & $22 \%$ & 0 & $0 \%$ & PDIA2 \\
\hline Y18D10A.11 & 7 & $28 \%$ & 0 & $0 \%$ & 7 & $30 \%$ & 0 & $0 \%$ & N/A \\
\hline RAB-7 & 5 & $32 \%$ & 0 & $0 \%$ & 4 & $24 \%$ & 0 & $0 \%$ & RAB7A \\
\hline $\mathrm{ABCF}-2$ & 9 & $18 \%$ & 0 & $0 \%$ & 3 & $7 \%$ & 0 & $0 \%$ & $\mathrm{ABCF} 2$ \\
\hline UGT-62 & 7 & $15 \%$ & 0 & $0 \%$ & 2 & $6 \%$ & 0 & $0 \%$ & UGT3A2 \\
\hline SPTL-1 & 2 & $7 \%$ & 0 & $0 \%$ & 6 & $19 \%$ & 0 & $0 \%$ & SPTLC1 \\
\hline VPR-1 & 4 & $25 \%$ & 0 & $0 \%$ & 4 & $33 \%$ & 0 & $0 \%$ & VAPB/ALS8 \\
\hline T09B4.2 & 4 & $15 \%$ & 0 & $0 \%$ & 3 & $15 \%$ & 0 & $0 \%$ & LAP1/LULL1 \\
\hline SUN-1 & 4 & $12 \%$ & 0 & $0 \%$ & 3 & $9 \%$ & 0 & $0 \%$ & SUN1 \\
\hline SEC-61 & 3 & $7 \%$ & 0 & $0 \%$ & 4 & $9 \%$ & 0 & $0 \%$ & SEC61A2 \\
\hline CYK-7 & 2 & $6 \%$ & 0 & $0 \%$ & 4 & $22 \%$ & 0 & $0 \%$ & N/A \\
\hline K08F4.3 & 3 & $29 \%$ & 0 & $0 \%$ & 3 & $29 \%$ & 0 & $0 \%$ & SSR2 \\
\hline EMR-1 & 3 & $22 \%$ & 0 & $0 \%$ & 2 & $13 \%$ & 0 & $0 \%$ & LEMD1 \\
\hline FAT-1 & 2 & $6 \%$ & 0 & $0 \%$ & 3 & $6 \%$ & 0 & $0 \%$ & FADS \\
\hline NKB-1 & 3 & $14 \%$ & 0 & $0 \%$ & 2 & $10 \%$ & 0 & $0 \%$ & ATP1B1 \\
\hline NPP-22 & 2 & $2 \%$ & 0 & $0 \%$ & 3 & $5 \%$ & 0 & $0 \%$ & NDC1 \\
\hline STT-3 & 2 & $3 \%$ & 0 & $0 \%$ & 3 & $5 \%$ & 0 & $0 \%$ & SST3 \\
\hline ZK686.3 & 3 & $9 \%$ & 0 & $0 \%$ & 2 & $6 \%$ & 0 & $0 \%$ & MAGT1 \\
\hline EMC-1 & 2 & $3 \%$ & 0 & $0 \%$ & 2 & $4 \%$ & 0 & $0 \%$ & EMC1 \\
\hline GEI-18 & 2 & $12 \%$ & 0 & $0 \%$ & 0 & $0 \%$ & 0 & $0 \%$ & LAP1/LULL1 \\
\hline
\end{tabular}


bioRxiv preprint doi: https://doi.org/10.1101/2020.04.22.055988; this version posted April 23, 2020. The copyright holder for this preprint (which was not certified by peer review) is the author/funder, who has granted bioRxiv a license to display the preprint in perpetuity. It is made available under aCC-BY-NC-ND 4.0 International license.

\section{table S1. Proteins that co-purify with OOC-5.}




\begin{tabular}{|c|c|c|c|c|}
\hline Strain & $\mathrm{T}\left[{ }^{\circ} \mathrm{C}\right]$ & Total eggs laid & Embryonic lethality [\%] & Male incidence [\%] \\
\hline \multirow[t]{6}{*}{ N2 } & 15 & $294.9 \pm 36.3$ & $0.2 \pm 0.2$ & $0 \pm 0$ \\
\hline & & $\mathrm{n}=10$ & $\mathrm{n}=2949$ & \\
\hline & 20 & $290.3 \pm 28.6$ & $0.7 \pm 0.6$ & $0.1 \pm 0.17$ \\
\hline & & $\mathrm{n}=19$ & $\mathrm{n}=5515$ & \\
\hline & 25 & $288.4 \pm 33.7$ & $0.29 \pm 0.38$ & $0.11 \pm 0.22$ \\
\hline & & $\mathrm{n}=9$ & $n=2596$ & \\
\hline \multirow[t]{4}{*}{ ooc-5(tn1757) } & 15 & $81.9 \pm 23.4$ & $99.2 \pm 1.1$ & ND \\
\hline & & $\mathrm{n}=30$ & $\mathrm{n}=2457$ & \\
\hline & 20 & $82.6 \pm 20.8$ & $98.8 \pm 1.8$ & ND \\
\hline & & $\mathrm{n}=35$ & $n=2892$ & \\
\hline ooc-5(tn1758) & 15 & $95.7 \pm 61.9$ & $19.5 \pm 14.4$ & $0 \pm 0$ \\
\hline \multirow[t]{5}{*}{ OOC-5::GFP } & & $\mathrm{n}=31$ & $n=2967$ & \\
\hline & 20 & $64.1 \pm 55.4$ & $50.6 \pm 29.8$ & $0 \pm 0$ \\
\hline & & $\mathrm{n}=29$ & $\mathrm{n}=1860$ & \\
\hline & 25 & $57.8 \pm 39.3$ & $80.6 \pm 33.3$ & $0 \pm 0$ \\
\hline & & $\mathrm{n}=30$ & $\mathrm{n}=1733$ & \\
\hline
\end{tabular}

\section{table S2. Brood sizes.}

Hermaphrodites were incubated at the indicated temperature (T) throughout their development. Then they were individually selected at the mid-L4 stage and transferred to new plates every $24 \mathrm{~h}$ over the course of 3-4 days. Plates were scored for dead embryos, surviving progeny, and males. Embryos not hatching within $24 \mathrm{~h}$ after being laid were considered dead. 


\begin{tabular}{|c|c|c|c|}
\hline Genotype & $\begin{array}{l}\text { Mean lifespan after mid-L4 }{ }^{\mathrm{a}} \\
{[\text { days } \pm \mathrm{SE}]}\end{array}$ & $\mathrm{n}$ & censored \\
\hline Wildtype & $19.24 \pm 0.29$ & 140 & 16 \\
\hline ooc-5(tn1757) & $17.3 \pm 0.35$ & 80 & 7 \\
\hline 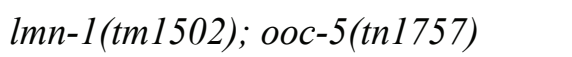 & $14.41 \pm 0.32$ & 120 & 44 \\
\hline $\operatorname{lmn}-1(\operatorname{tm} 1502) ;$ ooc-5(tn1757)/mIn1 & $12.07 \pm 0.19$ & 125 & 14 \\
\hline $\operatorname{lmn}-1(\operatorname{tm} 1502) ;+\operatorname{lm} \operatorname{In} 1$ & $10.36 \pm 0.28$ & 118 & 8 \\
\hline $\operatorname{lmn}-1(\operatorname{tm} 1502)$ & $9.8 \pm 0.18$ & 119 & 9 \\
\hline
\end{tabular}

\section{table S3. Loss of ooc-5 extends the lifespan of $\operatorname{lmn}-1$ mutants.}

The Wilcoxon tests of individual pairs comparisons for all the genotypes had a $\mathrm{p}<0.001$, except the $\operatorname{lmn}$ - 1 (tm1502) vs lmn-1(tm1502); +/mIn1 pair; which had a $\mathrm{p}=0.119 .{ }^{\mathrm{a}}$ For Kaplan-Meier survival curves see Fig. 1E. 


\author{
Strain Genotype \\ N2 Wild type, Bristol isolate \\ CA1219 ieSi21[sun-1p::sun-1::mruby::sun-13'UTR+Cbr unc-119] IV \\ DG4309 ooc-5(tn1757)/mIn1[dpy-10(e128)mIs14] II backcrossed x 6 \\ DG4377 ooc-5(tn1758[ooc-5::gfp::tev::3xflag:"HFDDEL” (endogenous ER retention motif)] II backcrossed \\ $\mathrm{x} 3$ \\ DG4463 npp-12(tn1780 [npp-12::gfp::tev::3xflag]) I; ooc-5(tn1757)/mIn1[dpy-10(e128)mIs14] II \\ DG4460 npp-12(tn1780[npp-12::gfp::tev::3xflag]) I \\ DG4504 lmn-1(tm1502)/tmC18[dpy-5(tmIs1236)] I; ooc-5(tn1757)/mIn1 [dpy-10(e128)mIs14] II \\ DG4505 Imn-1(tm1502)/tmC18[dpy-5(tmIs1236)] I; mIn1[dpy-10(e128)mIs14]/+ II \\ DG4543 Imn-1(tm1502)/tmC18[dpy-5(tmIs1236)] I; mIn1[dpy-10(e128)mIs14]/+ II; ieSi21[sun-1::mRuby] \\ IV \\ DG4544 Imn-1(tm1502)/tmC18[dpy-5(tmIs1236)] I; ooc-5(tn1757)/mIn1[dpy-10(e128)mIs14] II; ieSi21[sun- \\ $1:: m R u b y] \mathrm{IV}$ \\ DG4554 ooc-5(tn1757)/mIn1[dpy-10(e128)mIs14] II; npp-22(tn1794) V \\ DG4557 npp-22(tn1794[gfp::3xflag::npp-22]) V backcrossed x3 \\ DG4708 Imn-1(tm1502)/tmC18[dpy-5(tmIs1236)] I; sun-1(gk199)/tmC12[egl-9(tmIs1194)] V \\ DG4709 Imn-1(tm1502)/tmC18[dpy-5(tmIs1236)] I; sun-1(jf18)/tmC12[egl-9(tmIs1194)] V \\ DG4710 ooc-5(tn1757)/mIn1[dpy-10(e128)mIs14] II; sun-1(ff18)/tmC12[egl-9(tmIs1194)] V \\ DG4711 ooc-5(tn1757)/mIn1[dpy-10(e128)mIs14] II; tmC12[egl-9(tmIs1194)]/+ V \\ DG4712 ooc-5(tn1757)/mIn1 [dpy-10(e128)mIs14] II; sun-1(gk199)/tmC12[egl-9(tmIs1194)] V \\ DG4713 mIn1[dpy-10(e128)mIs14]/+ II; sun-1(jf18)/tmC12[egl-9(tmIs1194)] V \\ DG4714 mIn1[dpy-10(e128)mIs14]/+ II; sun-1(gk199)/tmC12[egl-9(tmIs1194)] V \\ DG4721 Imn-1(tm1502)/tmC18[dpy-5(tmIs1236)] I; zyg-12(or577) II \\ DG4755 ooc-5(tn1757)/mIn1[dpy-10(e128)mIs14] II; ribo-1(tn1891) IV \\ DG4759 Imn-1(tm1502)/tmC18[dpy-5(tmIs 1236)] I; zyg-12(ct350)/mIn1 [dpy-10(e128)mIs14] II
}


DG4760 Imn-1(tm1502)/tmC18[dpy-5(tmIs1236)] I; zyg-12(ct350)/mIn1 [dpy-10(e128)mIs14] II; itIs37; itIs 38

DG4786 ojIs9[zyg-12(all)::gfp]; ieSi21[sun-1::mRuby] IV

DG4572 Imn-1(tm1502)/tmC18[dpy-5(tmIs1236)] I; npp-22(tn17940) V

DG4789 ooc-5(tn1757)/mIn1[dpy-10(e128)mIs14] II; jIs9[zyg-12(all)::gfp+unc-119(+)]; ieSi21[sun$1:: m R u b y] \mathrm{IV}$

DG4535 lmn-1(tm1502)/tmC18[dpy-5(tmIs1236)] I; ooc-5(tn1757)/mIn1[dpy-10(e128)mIs14] II; itIS37[pie1p::mCherry ::H2B::pie-1 3'UTR + unc-119(+)] IV; itIS38[pie-1p::GFP::PH(PLCldelta1) + unc$119(+)]$

DG4541 Imn-1(tm1502)/tmC18[dpy-5(tmIs1236)] I; mIn1[dpy-10(e128)mIs14]/+ II; itIS37[pie1p::mCherry::H2B::pie-1 3'UTR + unc-119(+)] IV; itIS38[pie-1p::GFP::PH(PLCldeltal) + unc$119(+)]$

DG4950 lmn-1(tm1502)/tmC18[dpy-5(tmIs 1236)] I; ooc-5(it145)unc-4(e120)/mIn1 [dpy-10(e128)mIs14] II; itIS37[pie-1p::mCherry::H2B::pie-1 3'UTR + unc-119(+)] IV; itIS38[pie-

$1 p:: G F P:: P H(P L C 1$ delta1 $)+$ unc-119(+)]

DG4951 lmn-1(tm1502)/tmC18[dpy-5(tmIs 1236)] I; ooc-5(tn1709)/mIn1[dpy-10(e128)mIs14] II; itIS37[pie1p::mCherry::H2B::pie-1 3'UTR + unc-119(+)] IV; itIS38[pie-1p::GFP::PH(PLC1delta1) + unc$119(+)]$

table S4. Strains used in this study. 
table S5. Oligonucleotides used in this study.

movie S1. Movement of SUN-1 foci in early prophase of wild-type animals. 1-day old adult hermaphrodites carrying the SUN-1::mRuby expressing transgene were anesthetized and mounted for observation. Z-series were acquired every $15 \mathrm{~s}$ for 6-7 min. Maximum intensity projections were converted to grayscale and their LUTs were inverted.

movie S2. Movement of SUN-1 foci in early prophase of ooc-5(-) homozygous animals. 1day old adult hermaphrodites homozygous for the ooc-5(tn1757) deletion and carrying the SUN1::mRuby expressing transgene were anesthetized and mounted for observation. Z-series were acquired every $15 \mathrm{~s}$ for 6-7 min. Maximum intensity projections were converted to grayscale and their LUTs were inverted.

movie S3. Movement of SUN-1 foci in early prophase of ooc-5(-)/+ heterozygous animals. 1day old adult hermaphrodites heterozygous for the ooc-5(tn1757) deletion over a balancer chromosome (mInl) and carrying the SUN-1::mRuby expressing transgene were anesthetized and mounted for observation. Z-series were acquired every $15 \mathrm{~s}$ for 6-7 min. Maximum intensity projections were converted to grayscale and their LUTs were inverted. 